\title{
Article \\ Impact of Different Types of Head-Centric Rest-Frames on VRISE and User Experience in Virtual Environments
}

\author{
Andrej Somrak *®D, Matevž Pogačnik and Jože Guna \\ Faculty of Electrical Engineering, University of Ljubljana, Tržaška Cesta 25, 1000 Ljubljana, Slovenia; \\ matevz.pogacnik@fe.uni-lj.si (M.P.); joze.guna@ltfe.org (J.G.) \\ * Correspondence: andrej.somrak@ltfe.org; Tel.: +386-1-476-8732
}

Citation: Somrak, A.; Pogačnik, M.; Guna, J. Impact of Different Types of Head-Centric Rest-Frames on VRISE and User Experience in Virtual Environments. Appl. Sci. 2021, 11, 1593. https://doi.org/10.3390/ app11041593

Academic Editor: Ilsun Rhiu Received: 18 December 2020 Accepted: 5 February 2021 Published: 10 February 2021

Publisher's Note: MDPI stays neutral with regard to jurisdictional claims in published maps and institutional affiliations.

Copyright: (c) 2021 by the authors. Licensee MDPI, Basel, Switzerland. This article is an open access article distributed under the terms and conditions of the Creative Commons Attribution (CC BY) license (https:// creativecommons.org/licenses/by/ $4.0 /)$.

\begin{abstract}
This paper presents the results of a user study of the effects of different head-centric rest-frames on Virtual Reality-Induced Symptoms and Effects (VRISE) and the user experience in virtual environments (VE). Participants played the custom-designed 3D game in two different game modes (high action and low action). For assessing VRISE levels, we used the Simulator Sickness Questionnaire (SSQ) and Fast Motion Sickness Score (FMS). The presence was evaluated by SPES (Spatial Presence Experience Scale), and for the user experience, the short version of the User Experience Questionnaire (UEQ-S) was used. The results indicate that the usage of head-centric rest-frames negatively affected VRISE levels (more sickness) in the low action mode of the game. However, for the users experienced with VR technology, the VRISE disorientation symptoms were alleviated in a high action mode of the game with rest-frame glasses. We found no negative effect of rest-frames on the user experience and presence, except for some negative impact when using rest-frame glasses in the low action mode of the game. No negative impact on the performance itself was observed. That means that the usage of head-centric rest-frames is suitable for usage in VR applications. In terms of VRISE levels, we found out that rest-frame glasses are more suitable for the wearers of the distance spectacles, and a baseball hat is more suitable for non-wearers of distance spectacles.
\end{abstract}

Keywords: virtual reality; rest-frame; VRISE; VR sickness; cybersickness; user experience; presence; user study

\section{Introduction}

Although virtual reality technology (VR) has achieved amazing development and has reached technological maturity since 2012, when the so-called second wave of VR in 2012 began with the announcement of Oculus Rift, there are still some major drawbacks for technology acceptance, and broader user adoption are the health-oriented effects this technology has on humans.

Terms such as VR sickness, Cybersickesss, VRISE (VR-induced symptoms and effects), VIMS (visually induced motion sickness), simulator sickness, etc., describe those side effects and are often used interchangeably. In the present paper, we use the term VRISE to describe the side effects of VR usage. VRISE manifests in feelings of dizziness, disorientation, eyestrain, fatigue, nausea, etc., and it manifests during and after exposure to a virtual environment (VE). Sometimes, it causes side effects that can last for a prolonged time (hours, even days) [1]. Those side effects are disembarkment syndrome, recurrence of travel sickness, troubled hand-eye coordination, worsened vestibulo-occular reflex, and postural instability. VRISE also has a negative aftereffect on cognitive performance [2,3]. Studies have shown that $30 \%$ to over $80 \%$ of users experience side effects from VR usage [4]. VRISE symptoms are polysymptomatic (many symptoms) and polygenic (manifested symptoms differ from individual to individual) [4]. The effect of VRISE on users also affects technology acceptance, since experiencing side effects from VR usage, users would not be motivated to use the technology in the future or even recommend its usage to others. 
According to LaValle [5], VR is defined as a technology that induces targeted behavior in an organism by using artificial sensory stimulation, while the organism has little or no awareness of the interference. VR technology stimulates multiple senses and, assisted by multimodal interactions, creates the illusion of presence in VE. The human brain must integrate real-time vision, hearing, vestibular, and proprioceptive inputs to produce the compelling and captivating feeling of immersion in a VE [6]. In VR, there is no external force that influences vestibular stimulation, and there should be at least one mechanism for visual motion control that results in concordant visual and vestibular information [7]. Similar to the VR are augmented (AR) and mixed reality (MR) technologies, but they use a different approach. While VR aims to substitute virtual for real stimulation for one or many sensory organs, the AR and MR blends real and virtual stimulations [8]. The difference between $\mathrm{AR}$ and $\mathrm{MR}$ is that, in AR, the real world remains central to the experience, enhanced with digital information overlay, and, in MR, real and virtual worlds are intertwined, which allows for interaction and manipulation of both the physical and virtual environments. Through the usage of the Simultaneous Localization and Tracking (SLAM) technique, the space around the user is recognized, which allows for the virtual (digital) objects to be integrated into and responsive to the real world.

Experiencing VR is possible with head-mounted displays (HMD), projector-based immersive rooms (CAVE or C-Automatic Virtual Environment), and large monitors that can also provide an interesting immersive experience. A Taxonomy of current VR display devices is given in [9]:

- Portable HMD devices:

o With a mobile phone as a display and processing unit (Samsung Gear VR, Google Cardboard, Google Daydream, Zeiss VR One etc.)

o Stand-alone (All-In-One) portable VR devices (Oculus Go, Oculus Quest 2, Lenovo/Google Mirage Solo, Pico Neo 2/G2 etc.)

- Wired HMD devices:

o With a wired connection to a powerful computer for their operation (Oculus Rift S, HTC Vive Cosmos, Valve Index, OSVR, Windows Mixed Reality devices, Varjo VR etc.)

o Devices that connect to the gaming console (Sony PlayStation VR)

- Immersive rooms-CAVE (C-Automatic Virtual Environment)

- Large monitors

Portable VR devices that use a mobile phone as a display and processing unit are being taken off the market, and current development is focused on portable stand-alone HMD devices and wired HMD devices, which offers greater graphic fidelity over the portable devices. The wired HMD device is connected (with a cable or special wireless adapter) to the powerful computer, where all CPU and GPU processing is being made. The HMD serves as a display device and primarily consists of high spatial and temporal resolution (dual) displays, allowing for high-fidelity stereoscopic image rendering [10]. Sound is being played with the integrated binaural speakers or by the connected headphones. HMD devices also have integrated hand/body and space (for room-scale experience) tracking and, with some devices, even eye-tracking. Interacting in the virtual environment is possible with the complementary controllers. Some newer devices also utilize hand-tracking and voice recognition as an option.

While first generations of VR HMD devices had only three-degrees of freedom (dof) tracking capabilities, limited resolution, and field-of-view, high and noticeable motionto-photons latency, tracking latency, flicker, etc., the latest commercially available devices have built-in state-of-the-art technology, which includes fast and pixel-dense displays; advanced motion-tracking (head, eyes, hands, and body); artificial intelligence; etc. The latest devices have built-in inside-out tracking systems with integrated cameras, without external sensors, where the setup sometimes could be cumbersome and not suitable for easy transportation. Still, the problem with VRISE was only partially solved with technology 
improvements, as the device is one of the three factor clusters that have an effect on VRISE (besides individual and task factors) $[6,11]$. Since individual factors cannot be overcome, the main focus of developing VR experiences with no or minimal side effects should be on task-related factors.

When this study was performed and the results were analyzed, it was during the time of Coronavirus disease 2019 (COVID-19) lockdowns, social distancing, and travel restrictions. The usage of VR technology is extremely helpful in a situation like this. It helps people virtually and immersively discover the world, virtually connect with friends and family, and that way, the time of being at home most of the time passes easier. VR was already proven as a helpful medical tool and is used to alleviate depression and boost moods, which is a common side effect of staying at home and not socializing for a prolonged time. VR technologies are used in many fields, for home and business uses, education, training, entertainment, industrial design, architecture, etc., and can increase the human-computer bandwidth.

There are multiple theories of why VRISE does occur, and more than 40 factors have been discovered [12] that influence VRISE. Our study is based on the Rest-Frame Hypothesis (RFH) [13], which offers an alternate theory on motion sickness, where the emphasis is on the role of spatial-perceptual references affected by reference and rest-frames.

A Reference Frame is a coordinate system with respect to which positions, orientations, and motions can be judged. Rest-Frame is the particular reference frame that a given observer takes to be stationary [14] and judges other motions relative to. Rest-frames can be defined as the vertical references provided by visual and idiotropic vertical cues, which are relatively stationary to subjects [15]. The rest-frame is considered one of several reference frames that the nervous system has access to and provides the observer with spatial information of stationary objects [16]. Under normal conditions, one of these is selected by the nervous system as the comparator for spatial judgments (selected restframe). In some cases, the nervous system is not able to choose a single rest-frame [14]. An egocentric rest-frame is centered on the navigator, whereas an allocentric rest-frame is centered externally in the environment and defined by features of the environment [17]. Egocentric rest-frames are also called player-fixed rest-frames and are defined with respect to the person and are required for the current state of VR technology, which allows players to look around. Earth-fixed (allocentric) rest-frames start to move if the subject is looking around in VR, and it might disappear and, thus, not be available as a rest-frame anymore. Player-fixed rest-frames might be challenging in regard to occluding or being occluded by other objects in the scene [18]. Egocentric rest-frames can be divided into body-centric, head-centric, and oculo-centric [13]. In the virtual environment (VE), the rest-frames are integrated into the scene, remain fixed in relation to the real world or body part, and do not move as the user or the body part virtually moves. Rest-frames can be utilized as backgrounds, and also, smaller foreground cues can help to serve as a rest-frame.

The Rest Frame Hypothesis states that sickness does not directly arise from conflicting orientation and motion cues but, rather, from conflicting rest-frames implied by those cues [19]. What is crucial is not the full set of cues in an environment but, rather, how those cues are interpreted to influence one's sense of what is and is not stationary. Sickness is inextricably tied to one's internal mental model of what should be stable. Although this is only a slight refinement to sensory conflict theory [20], it suggests that attempts to reduce sickness may usefully focus on the particular stimuli that influence the selected rest-frame rather than on all orientation and motion stimuli. The RFH provides an explanation for the occurrence of VRISE and, also, an approach to its instant reduction. The RFH assumes the brain has an internal mental model of which objects are stationary and which are moving. When new incoming sensory motion cues do not fit the current mental model of the restframe, or the subject has difficulty in selecting a consistent rest-frame, sickness results. The rest-frame must remain congruent with inertial and visual cues. The RFH allows for the existence of sensory conflicts without causing motion sickness if those conflicting cues are not essential to the rest-frame's stability. The RFH predicts simulator sickness from 
the dependency on the match between the selected rest-frame and the subject's motion. Prothero and Parker [13] also suggested that motion sickness occurs when there are too many rest (reference)-frames to choose from, and the individual becomes confused and conflicted. A possible way to combat motion sickness, in this case, would be to introduce one rest-frame, an independent visual background (IVB), salient enough to attenuate focus from other competing and conflicting rest-frames [21]. The rest-frame salience is an important factor, but a study by Weinrich et al. [18] showed no significant impact of the rest-frame salience or on the VRISE levels or on the game experience.

VRISE is much less of an issue for optical see-through augmented reality HMDs, because users can directly see the real world, which acts as a rest-frame consistent with vestibular cues. VRISE is also reduced when the real world can be seen in the periphery of the outside edges of an HMD.

An independent visual background (IVB) is a visual scene made to appear behind the content-of-interest of a virtual environment and controlled independently from the contentof-interest [14]. It is a different kind of rest-frame and has been shown to be effective in alleviating VRISE [15]. A visual scene can be divided into components, including one labeled the "content-of-interest" and another called the "independent visual background". IVB could provide visual motion and orientation cues that match those from the vestibular system, which inclusion in a VE should reduce sickness [22].

Presence in a VE, the vivid feeling of being in, or the ability to interact with the VE can be enhanced when the user perceives the rest-frame to be a part of the virtual world instead of the real world [21]. This is suggested by a present hypothesis [13], which states: The sense of presence in an environment reflects the degree to which that environment influences the selected rest-frame. That is, presence in a virtual environment is related to the VE's ability to influence the sense of position, angular orientation, and motion. The experiment performed by Ricke et al. [23] supported the hypothesis that top-down or cognitive influences do play a considerable role in self-motion perception. Presence should be indicated by the relative influence on the subject's motion perception of virtual as opposed to real rest-frame cues-that is, the degree to which virtual cues overwhelm real cues [3].

This paper presents the influence of head-centric rest-frames on VRISE and user experiences in VE. In our study, the participants played a custom-designed 3D game with different scenes (forest, ancient desert, and village) and different types of head-centric rest-frames. One of the head-centric rest-frames was in central vision (glasses), and the other one was in peripheral vision (baseball hat). We analyzed the effects in two different game modes (high action and low action).

The results showed that the usage of head-centric rest-frames negatively affected VRISE levels (more sickness) in the low action mode of the game. However, for the users experienced with VR technology, the VRISE disorientation symptoms were alleviated in a high action mode of the game when the rest-frames glasses were used. Rest-frames did not affect the user experience, except in the low action mode of the game, where the pragmatic quality was negatively affected by the usage of rest-frame glasses. The presence was only affected (less presence) when rest-frame glasses were used in the low action (LA) mode of the game. Additionally, no effect on the performance (obtained scores and completion time) was observed with the usage of rest-frames for both modes of the game. We did not find any differences between both types of rest-frames in terms of user experience, presence, and performance. In terms of VRISE levels, we found out that rest-frame glasses are more suitable for the users who are wearing distance spectacles and a baseball hat for non-wearers of distance spectacles.

We compared the modes of the game in terms of VRISE, user experience, presence, and performance. We found significantly fewer disorientation symptoms and significantly fewer sickness symptoms assessed by the Fast Motion Sickness (FMS) Questionnaire for the LA mode of the game. For user experiences, it was found out that a better experience happened in the high action (HA) mode of the game for the short version of the User Experience Questionnaire (UEQ-S) Overall scores, for the UEQ-S Hedonic Quality scores, 
and the Virtual Reality Neuroscience Questionnaire (VRNQ)-User Experience. UEQ-S Pragmatic Quality subscale data showed that the UEQ-S Pragmatic Quality scores were significantly lower in the HA mode of the game. The results showed that, although the VRISE levels were higher in the HA mode of the game, the user experience was better, except for the pragmatic quality of the user experience. An impact on presence was found only between rest-frame conditions (higher presence) for the Spatial Presence Experience Scale (SPES) Total scores, as well as for the SPES Self-location and SPES Possible Actions subscales.

The key contributions of this paper are:

- a confirmed suitability of head-centric rest-frames glasses for experienced VR users in provocative VR content $(\mathrm{H} 1)$,

- showing that head-centric rest-frames are not suitable for inexperienced VR users in nonprovocative VR content,

- $\quad$ showing the better suitability of rest-frame glasses on VRISE levels for users who are wearing distance spectacles ( $\mathrm{H} 2)$,

- $\quad$ showing the better suitability of a baseball hat as a rest-frame for non-wearers of distance spectacles $(\mathrm{H} 2)$,

- $\quad$ showing no effect of rest-frames on the user experience and presence, except negative effects when using rest-frame glasses in the LA mode of the game (H3),

- confirming no effect of the rest-frames on performance (H5),

- confirming a negative effect on VRISE levels in more provocative VR contents (H6),

- confirming a positive effect on the user experience in more provocative VR contents (H7),

- confirming a positive effect on presence in more provocative VR contents (H8), and

- the quality of VR software assessments by the novel VRNQ Questionnaire.

\section{Background and Related Works}

In this section, we will review relevant related works. We reviewed works related to the research of rest and reference frames in VR applications.

In a study by Wienrich et al. [18], the authors analyzed the impact of a virtual nose (head-centric) rest-frame on VR sickness and game experience in a virtual reality jump'n'run game. This was the only previous study that analyzed the effects of head-centric rest-frames in virtual reality. They found out that using a virtual nose reduced VR sickness, while it did not affect the game experience. They also investigated the rest-frame salience's significance, which showed no significant impact, either on simulator sickness or on the game experience. They concluded that a rest-frame in the form of a virtual nose could be used in virtual reality applications to reduce VR sickness.

Cao et al. [15] analyzed the effects of static and dynamic rest-frames on VRISE, assessed by the Simulator Sickness Questionnaire (SSQ) and discomfort level. As a rest-frame, they used a see-through metal net surrounding users above and below their seat, which was kept stationary relative to the real world. The rest-frame moved virtually with the cockpit and did not move with the user's head or virtual motion. The participants were seated in a physical chair and also virtually seated in a cockpit while navigating through the virtual environment. While static rest-frames have fixed opacities, dynamic rest-frame opacities change in response to visually perceived motion as users virtually traverse the VE. The scale of discomfort level ranged from 0 to 10 ( 0 being most comfortable and ten being the most uncomfortable). The participants reported their discomfort levels six times during the gameplay while passing through a waypoint. The results showed that a VE with a static or dynamic rest-frame allowed users to travel through more waypoints before stopping due to discomfort compared to a virtual environment without a rest-frame. Further, a virtual environment with a static rest-frame was also found to result in more real-time reported comfort than when there was no rest-frame.

In the study of Nguyen-Vo et al. [17], the authors investigated the effects of simulated reference frames on spatial orientation. They used two different types (an allocentric reference frame-simulated room and an egocentric reference frame-simulated CAVE) of 
visually simulated reference frames in a navigational search task in a mixed-method study. The results showed that an allocentric reference frame significantly improved the user performance in navigational search time and overall travel distance, while an egocentric reference frame did not help significantly. They also analyzed the effect on VRISE using a single question method to assess the level of overall motion sickness. They found out that a simulated CAVE (body-centric rest-frame) significantly reduced the level of motion sickness compared to no reference frame.

Chang et al. [16] concluded the experiment where they investigated the effects of rest-frames on VRISE and oscillatory brain activity. In a roller coaster simulation, the participants experienced a rest-frame condition and a non-rest-frame condition. The restframe was presented as a grid of white lines, consisting of two horizontal and two vertical lines superimposed on the simulator's display. Based on the VRISE levels and EEG changes, they suggested that rest-frames may reduce or delay the onset of VRISE by alleviating users' attention or perception load.

An independent visual background (IVB) that never moves relative to the individual was utilized in a within-subjects design study by Duh et al. [24], where the subjects were exposed at two scene motion frequencies and three IVB conditions in a projection-based system. The rolling scene used was a cartoon scene, and over the entire scene, the IVB was superimposed at two brightness levels-dim and bright. At the dim level, the subjects were just able to detect the IVB, and at a bright level, the subjects were easily able to detect the IVB. For low-frequency scene movements, subjects exhibited less balance disturbances when the IVB was presented. The authors suggested that an IVB may alleviate disturbances when conflicting visual and inertial cues are likely to result in a simulator or VE sickness.

The effect of IVB on VRISE and presence was researched in a study by Lin et al. [22], where subjects were exposed to a complex motion on prerecorded trajectories through a simulated environment in a driving simulator in a projection-based system. There were three IVB conditions: grid (8 horizontal and 35 vertical grid lines), less cloud (seven clouds), and many clouds (28 clouds). Using the Revised Simulator Sickness Questionnaire (RSSQ), the subjects reported less nausea when the many clouds IVB was used relative to the grid IVB condition. The results indicated that a natural IVB composed of meaningful objects is more effective than a grid for alleviating VRISE levels. The results also showed that different types of IVBs do not significantly influence subjects' presence and enjoyment levels.

A virtual guiding avatar (VGA), which combines self-motion prediction cues and an IVB, was proposed in a within-subjects design study by Lin et al. [25] to alleviate the VRISE levels. The VGA was presented as an abstract airplane, and its purpose was to lead the participant along a horizontal motion trajectory through a VE. In a complex visual motion in a cartoon-like environment, the participants were exposed to a driving simulator. The VGA had three motion properties: fixed, rotation only, and rotation plus translation. The results showed that the VRISE levels were reduced when the VGA that presented rotational cues alone or rotation plus translation were utilized. Additionally, the VGA increased the participants' sense of presence and enjoyment.

An alternative to grid lines is to permit the participant to partially see the real environment behind the virtual environment. Prothero et al. [26] used a partially occluded HMD so the virtual room could be seen overlaid on the actual environment in two similar experiments. The primary difference was that the second experiment required focusing on the actual room. Their first experiment reported lower SSQ-T scores and fewer postural stance breaks in the independent visual background condition. The second experiment showed no difference with the condition on the SSQ-T but fewer stance breaks for the independent visual background condition. The differing results could mean the additional focus on the background or that the elimination of a participant from part of the analysis had an effect. The median score on the SSQ-T was identical between the two studies, but the standard deviation was different.

Prothero and Parker [13] showed that the IVB reduced self-reported simulator sickness, while it did not affect the vection illusion. 
Reviewing the background and related works shows the importance of rest-frames and their effects on VRISE, the user experience, and presence in the domain of VR. The RFH has been verified in VR, but most studies have been done in projection-based systems [15], using IVB or allocentric rest-frames. Stable rest-frames (RFs) have historically not been possible with HMDs due to the need for low latency and high-quality tracking and calibration. However, RFs can now be rather stable with modern VR technology [4]. Although RFH and the effects of rest-frames to alleviate VRISE levels is a proven concept, there are few studies that have analyzed the impact more deeply, with different kinds, sizes and shapes, and positions of rest-frames. More studies were done to assess the effects of rest-frames in virtual reality on spatial perception and navigation, and no study analyzed the impact of rest-frames on presence and performance. Therefore, we proposed head-centric rest-frames, which correspond to real objects in a real world, and participants are used to wearing them. This study extends the results of a study by Wienrich et al. [18], where the authors analyzed the impact of a head-centric rest-frame on VRISE and game experience in a VR game. They used a virtual nose for the rest-frame, which was in the lower part of the peripheral vision. Our study utilized glasses, which can be worn as distance spectacles or sunglasses, and a baseball hat-specifically, the shield of a baseball hat seen in the upper part of peripheral vision. Glasses were placed in the central vision and directly occluded the scene of a virtual environment.

To the authors' best knowledge, there is no study where the usage of head-centric rest-frames and analyzing their effects on VRISE, the user experience, and presence was researched in such a way.

\section{Research Questions and Hypothesis}

The main research questions in this study were:

- Do the head-centric rest-frames have an effect on the VRISE levels, user experience, presence, and performance?

- Is there a difference between the effects of different types of head-centric rest-frames based on their placement (of the central or peripheral vision) in terms of VRISE levels, user experience, presence, and performance?

- Are there differences between LA and the more provocative HA mode of the game in terms of the VRISE levels, user experience, and presence?

Based on the background research, we developed hypotheses that state (H1) that the head-centric rest-frames do positively affect VRISE levels that alleviate side effects from VR usage (less sickness). We state (H2) that there is a difference between the effects of different types of head-centric rest-frames in terms of the VRISE levels, user experience, presence, and performance. We state $(\mathrm{H} 3)$ that the head-centric rest-frames do not affect the user experience, (H4) have a positive effect on presence, and no effect on (H5) performance. We state (H6) that a more provocative HA mode of the game will have a negative effect on the VRISE levels (more sickness) compared to the LA mode of the game and have (H7) a positive effect on the user experience and $(\mathrm{H} 8)$ on presence.

\section{Method}

This section describes the study design with the emphasis on the participants, apparatus, software and game design, experiment procedure, and evaluation metrics used.

\subsection{Participants}

A total of 44 participants took part in this study. Twenty-seven of them were males, and 17 of them were females. Participants were, on average, 32.55 years old ( $\mathrm{SD}=8.15$ years), ranging from 19 years to 47 years. They came from mixed backgrounds: students, postgraduate researchers, academic staff, users interested in virtual reality, gamers, etc. Participants were recruited from the University of Ljubljana through invitations on web pages dedicated to gaming and virtual reality and invitations on social media to access the more general public. Only healthy participants were selected, whose participation was voluntary. 
Twenty participants had previous experience with VR devices. They reported they experienced virtual reality with Oculus Rift, HTC Vive, Oculus Go, Oculus Quest, Samsung Gear VR, Google DayDream, Windows Mixed Reality, and Sony Playstation VR. Fourteen of them previously noticed side effects due to VR usage. They reported general discomfort (1), tiredness (1), headache (2), eye strain (3), sweating (7), nausea (5), stomach awareness (2), paleness (1), dizziness (2), vertigo (5), disorientation (2), and postural instability (6). The previous experience with VR was a dichotomous variable based on the participants' responses to a question of whether they experienced VR previously.

Additionally, 17 of the participants were active gamers (based on activity in the last six months) —of which, 15 of them mainly played games on a computer, 6 of them on a video console, 11 of them on a mobile phone, and 1 of them on a tablet PC. Eleven participants used distance spectacles, and 13 participants were using contact lenses. All of them had normal vision or corrected-to-normal vision. Thirty-seven (84.09\%) participants were physically active, but none of them were into sports professionally.

Before taking part in the study, all participants provided written informed consent. No participant received any compensation for participation in the study.

\subsection{Apparatus}

The experiment was conducted using an Oculus Rift $\mathrm{S}$ head-mounted device (HMD). The HMD was connected to a high-performance gaming computer (CPU Intel core i7 7700 K, GPU Nvidia GTX 1070, RAM 16 GB DDR4 3000 MHz, SSD Samsung Evo 850). Wireless Oculus Touch controllers with 6 degrees of freedom were used for the navigation and interaction within the VE. Integrated Oculus Insight tracking without external sensors (inside-out tracking) was used for motion and controller tracking. Oculus Insight uses a combination of five cameras built into the HMD and information from the accelerometers in the HMD and controllers. It also exploits artificial intelligence to predict what path the controllers will most likely be taking when outside the cameras' field of view. The sound was played through a pair of speakers integrated into the headband.

Fulfilment of the questionnaires by the participants before, in the middle, and after the experiment was done on a notebook PC with a touch screen.

\subsection{Software and Game Design}

The participants played the custom-designed VR first-person game, which was developed in Unity. In the game, players had to find the way through the forest, ancient desert, and village (scenes) by collecting coins that were placed on the predetermined path. Each of the scenes was divided by sliding doors, which had to be opened by the player in order to proceed. The participants were asked to collect coins by passing through them, which made them disappear. The next coin to collect was always visible in the field-of-view so that participants stayed on the predetermined path and did not get confused about where to go forward. None of the participants had any problems staying on the path. Participants were also instructed to collect as many coins as possible (to collect all coins was not required to finish the level) and to finish the level as fast as possible, to not wander around and stay too long in each level. Each coin was animated and was rotating around its y-axis. The participants' virtual hands and controllers were drawn in a virtual environment. Extra caution was made when developing the game, so that playing and interacting would be easy and intuitive even for beginners and non-gamers.

Each collection of the coin was scored, and the current score and passed time was displayed on the screen, to be captured by the researcher after the participant completed the level. This information was not visible to the participants. It was only visible on the monitoring TV screen that would not affect the player during the gameplay and possibly serve as a rest-frame to the participant. The level was completed when the player passed through the finishing portal. There were also other action movements in the game, such as passing over a glass bridge over a deep channel, ascending and jumping off the building, and passing through a tunnel. 
There were two different modes of the games, a high action (HA) and a low action (LA) game. In a high action game with more provocative content, the forward-moving speed and jaw rotation speed was higher (walking vs. running), some of the coins were placed higher, so players have to jump to collect those coins (those coins were scored two points, while ordinary coins were scored one point). Additional jumping should contribute more to VRISE levels because of the extra vertical movement in the y-axis. Each of the modes of the game had three different conditions, with or without head-centric rest-frames. The comparison of all conditions is shown in Table 1. A forest scene from the low action game mode with rest-frame glasses is shown in Figure 1, an ancient desert scene from the high action game mode is shown in Figure 2, and a village scene from the low action game mode without rest-frames is shown in Figure 3.

Table 1. Comparisons of the conditions used in the study. The condition without the rest-frames is referred to as NORF, condition with rest-frames glasses as RFG, and condition with the baseball hat as RFH.

\begin{tabular}{cccccc}
\hline Condition & Mode of the Game & Translation & Rotation & Additional Actions & Rest-Frames \\
\hline LA_NORF & Low activity & Walking & Slow & No & No rest-frames \\
LA_RFG & Low activity & Walking & Slow & No & No \\
LA_RFH & Low activity & Walking & Slow & Shield of a baseball hat \\
HA_NORF & High activity & Running & Fast & Yes, jumping & No rest-frames \\
HA_RFG & High activity & Running & Fast & Yes, jumping & Glasses \\
HA_RFH & High activity & Running & Fast & Yes, jumping & Shield of a baseball hat \\
\hline
\end{tabular}

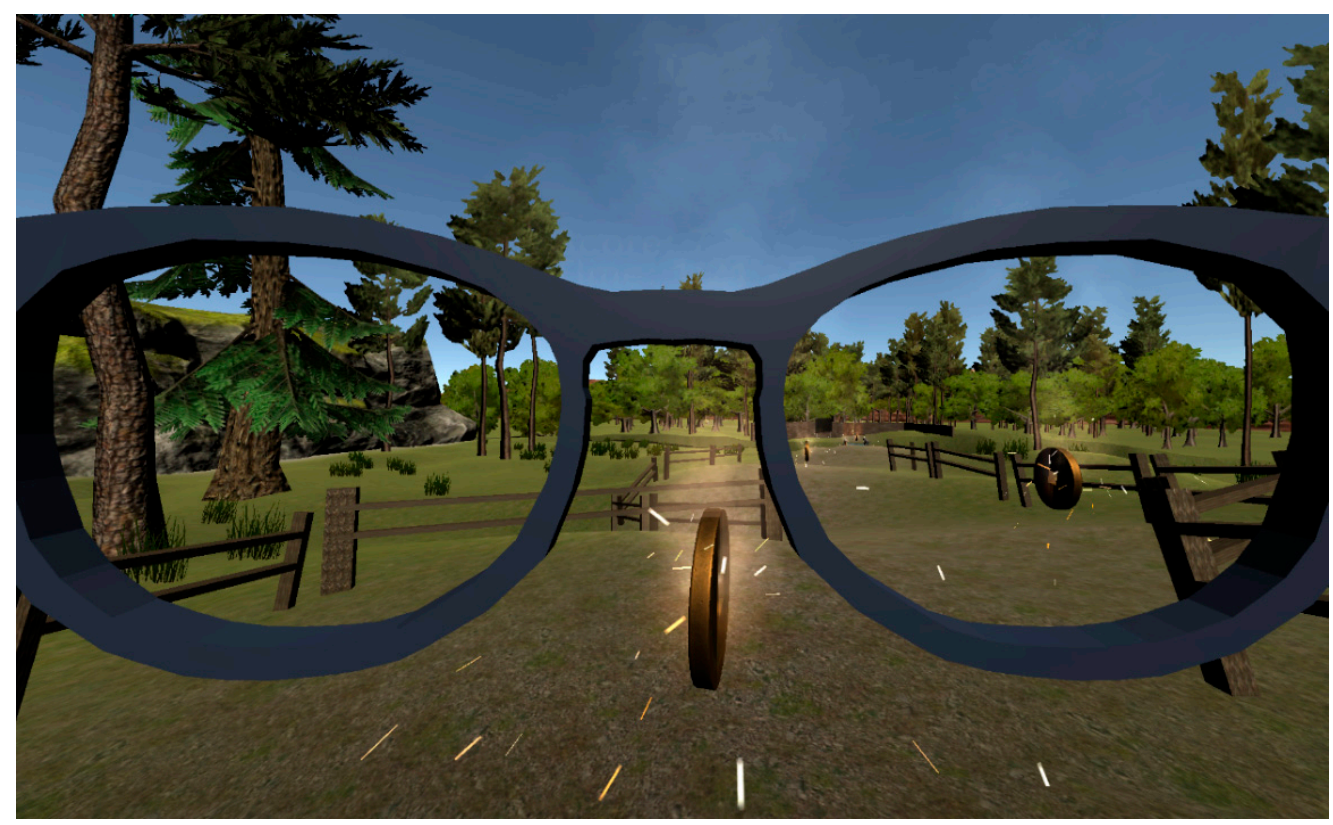

Figure 1. The forest scene in low action (LA) mode game with glasses as a head-centric rest-frame. Golden coins are set on the player's height, so there is no need to jump to collect them.

In a condition without a rest-frame, the participants played the game without any additional visual cues. This condition served as a baseline condition, which enabled us to compare them with other conditions with overlaid visual rest-frames. One of the head-centric rest-frames was in the central vision (glasses), and the other one was in the peripheral vision (baseball hat). Both rest-frames were natural, and the participants had experience with wearing them in a real-world environment. Being in the central vision, glasses were more salient than a baseball hat. Therefore, most participants noticed the glasses, whereas the baseball hat was noticed only by a few participants. For developers, a baseball hat, which was almost unnoticeable and did not cover the main plot area, is a 
better solution. Apart from that, both rest-frames used did not move, disappear, occluded, or were occluded themselves when players moved their heads in VR. They should help participants without distracting them from the game.

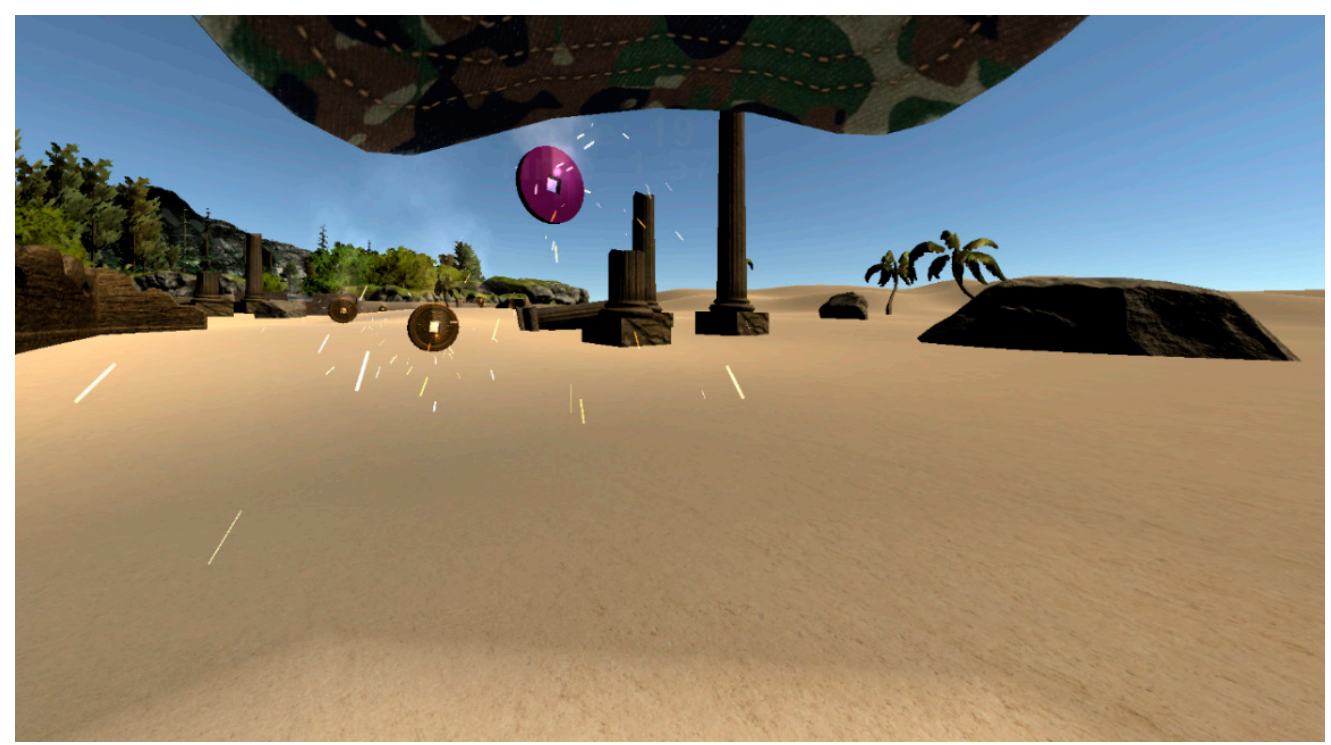

Figure 2. The ancient desert scene in the high action (HA) game with a baseball hat as a head-centric rest-frame. In the front, there is a visible violet coin. For collecting it, the player needed to jump.

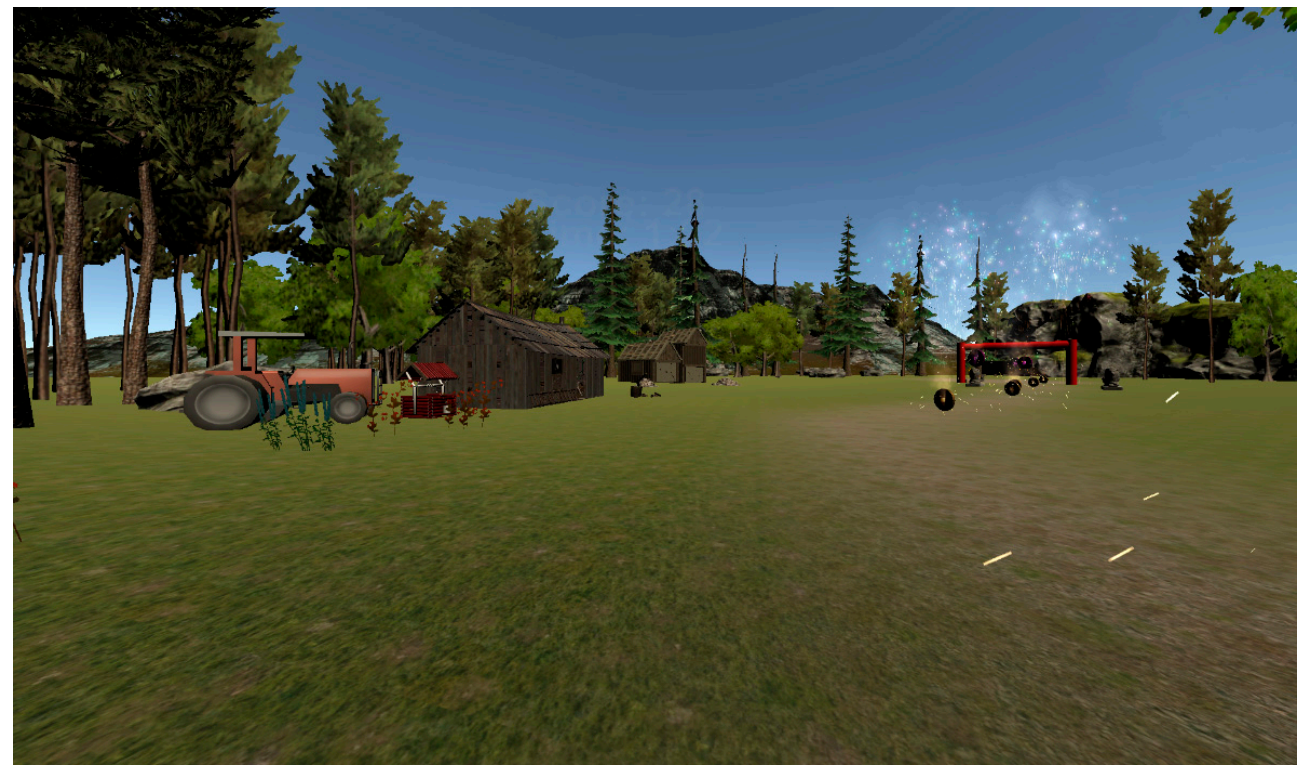

Figure 3. The village scene in the LA game without rest-frames. There is a visible red portal in the background that was needed to pass through to finish the game.

For locomotion, we used smooth artificial locomotion, a technique which is similar to the mechanics of traditional first-person shooters (FPS) played with a controller (using a trackpad or a thumbstick) or keyboard on a 2D display, where the jaw and translation is handled by a controller. This technique is known to induce more VRISE than other locomotion techniques and is one of the most used interactions and locomotion interfaces for VR devices. The perception of moving through a virtual environment as one is walking while one's body is stationary can induce VR sickness regarding sensory conflict theory, and it is a cause of vestibular mismatch (vestibular and visual cues of motion are in conflict), 
which can trigger dizziness and malaise. This locomotion method was selected for the game to efficiently determine the effect of head-centric rest-frames on VRISE.

An Oculus Touch controller was used as the navigation interface. A thumbstick on the controller was used to translate and rotate the participants' virtual avatars (tilting in the $y$-axis was used to freely move forward/backward, and tilting in the $\mathrm{x}$-axis was used to jaw rotate the participants' avatars). Any of the buttons $\mathrm{A}, \mathrm{B}, \mathrm{X}$, and $\mathrm{Y}$ were used to open the doors when the participant was near the door. The trigger button was used for jumping. The participants could freely look around by physically turning their heads while not moving in a virtual environment, whereas moving and rotating their head would move them in the head direction. Due to being seated, this was only possible for small corrections of the course. For more extensive rotations, a rotation with a controller was needed. Therefore, it was used a mixture of artificial controller rotation and physically turning the head for rotation.

The application was optimized to achieve a constant 80 frames per second (FPS) throughout all the levels. Eighty frames per second is the maximum refresh rate for the Oculus Rift $S$ device. There was no noticeable latency of tracking during the experiment, since the motion-to-photos latency is also a significant factor that affects the VRISE.

A notification sound was played via the speakers whenever a coin was collected successfully and when the door was opened to proceed to the next scene.

\subsection{Metrics}

In our study, we used existing, established standard methods (questionnaires). The usage of standard methods, which were already used and validated in other studies, also enabled comparing the results.

For assessing the VRISE levels, we used the Simulator Sickness Questionnaire (SSQ) [27], Fast Motion Sickness Score (FMS) [28], and a novel Virtual Reality Neuroscience Questionnaire (VRNQ) [29] — VRISE subscale. We used the Spatial Presence Experience Scale (SPES) [30] for assessing the presence. For the user experience, we used the short version of the User Experience Questionnaire (UEQ-S) [31] and the VRNQ Questionnaire-User Experience subscale.

The SSQ Questionnaire is the most widely used questionnaire in VR studies for assessing VRISE. The SSQ consists of 16 items, where participants give a score of 0 (none), 1 (slight), 2 (moderate), or 3 (severe) to 16 individual symptoms of VR Sickness. The SSQ can be administered before and after the virtual experience. The SSQ Questionnaire provides a total SSQ scale (SSQ-T), which consists of three subscales of nausea (SSQ-N), disorientation (SSQ-D), and oculomotor (SSQ-O). Nausea includes symptoms such as stomach awareness, increased salivation, and nausea itself. Oculomotor includes eyestrain, headache, and blurred vision, and disorientation includes symptoms such as dizziness, vertigo, and difficulty focusing. By combining scores from multiple symptoms, a score for each subscale is calculated. From these partial scores, the total SSQ score is calculated, where higher scores indicate greater VRISE levels. The scoring procedure was conducted in the manner recommended by Kennedy et al. [27].

FMS is a single-item verbal rating scale where participants gave a score from 0 (no sickness at all) to 20 (frank sickness) to evaluate the level of their sickness they felt in the virtual experience. In contrast to the SSQ Questionnaire, where the VRISE levels are measured before or after the virtual experience, FMS enables tracking sickness levels during the virtual experience and capturing its time course. Participants had to focus on nausea, general discomfort, and stomach problems and take these parameters into account when making their judgments. They were asked to ignore other possible distorting effects, such as nervousness, boredom, or fatigue.

UEQ-S contains eight items rated through the 7-stage Likert scale, measuring two dimensions (quality aspects) of user experience:

- Pragmatic Quality: described interaction qualities related to the tasks or goals the user aims to reach when using the product. 
- Hedonic Quality: did not relate to tasks and goals but described aspects related to pleasure or fun while using the product.

The items are scaled from -3 to +3 . Higher scores indicate greater levels of agreement with scales, while lower scores indicate greater levels of disagreement. Thus, -3 represents the most negative answer (fully agreeing with the negative term), 0 a neutral answer, and +3 the most positive answer (fully agreeing with the positive term). All scores above one are considered as a positive evaluation. We decided to use a short version of the UEQ Questionnaire, because the main focus of the study was not evaluating the user experience, consequently reducing the duration of questionnaires that had to be fulfilled. Besides that, we also included a VRNQ questionnaire, which also assessed user experience and is dedicated for use in virtual reality.

VRNQ is a novel questionnaire that assesses and reports both the quality of software features and VRISE intensity. It can be used to determine the quality of VR software in terms of user experience, game mechanics, in-game assistance, and VRISE. It is composed of four sections (User Experience, Game mechanics, In-game assistance, and VRISE), each section having five items rated through a 7-stage Likert scale, ranging from extremely low (1) to extremely high (7). VRNQ provides a total score corresponding to the overall quality of the VR software, as well as four subscores for each section/domain. The higher scores indicate a more positive outcome, which also applies to the evaluation of the VRISE levels. The minimum cut-offs indicate the lowest acceptable quality of VR software, while the parsimonious cut-offs indicate more robust VR software suitability. Compared to the SSQ Questionnaire, it also assesses software attributes, not just the symptoms pertinent to simulator sickness.

The spatial presence can be defined as a user's subjective feeling or conscious experience of "being there" in a mediated (computer-generated) environment [32], even when one is physically situated in another. It is not readily amenable to objective physiological definition and measurement. The presence is more convincing with more interactivity, immersion (reproduction of the conditions of the physical presence), and realism. SPES is a short and convenient-to-apply eight-item self-report measure. It is derived from a process model of spatial presence. It assesses spatial presence as a two-dimensional construct that comprises a user's self-location and perceived possible actions in a media environment. It provides two subscales (Self-Location and Possible Actions) and a total score of spatial presence. Four items are used per subscale. SPES can be applied to diverse media settings, ranging from immersive virtual reality to interactive audiovisual video game applications, noninteractive television, and even books. All items are phrased in a way that can be applied in a posttest of VE exposure.

\subsection{Experiment Environment}

The experiment took place in the Multimedia laboratory, which is set up as a living room. Experimenters had full control over the environmental variables. There were no external sources of noise that could interfere with the experiments. Environmental conditions were also monitored (temperature, humidity, and lighting conditions).

\subsection{Procedure}

The experiment was performed using a $2 \times 3$ repeated measures within-subjects design in which all participants experienced all six conditions in one session. The independent variables were mode of the game ( 2 levels: low action and high action mode) and simulated head-centric rest-frames (3 levels: no rest-frame, rest-frame glasses, and rest-frame baseball hat). The condition without the rest-frames is referred to as NORF, condition with rest-frames glasses as RFG, and condition with the baseball hat as RFH. Regarding the mode of the game, the condition of the low action mode of the game is referred to as LA, and the condition of the high action mode of the game is referred to as HA. The comparison of the conditions is shown in Table 1. 
We prepared five questionnaires to gather quantitative data. The first questionnaire was fulfilled online via a web browser at home before the experiment. After participants confirmed attending the experiment, a link was sent to the first questionnaire, which included their demographic data, sports activity level, vision and hearing, gaming, and VR technology experience. This questionnaire also included the Edinburgh Handedness Inventory-Short Form [33], Immersive tendencies questionnaire (ITQ) [34], Big five Personality Inventory-Short version (BFI-10) [35], and Motion Sickness Susceptibility Questionnaire Short Form. All participants were identified by a unique ID that was assigned to them. Completion of this questionnaire was mandatory for the participant to be able to attend the experiment. They were instructed not to consume any food, stimulant drinks, or alcohol two hours before the experiment.

When the experiment took place, the first step was to receive the participants and to welcome them at the Multimedia Laboratory. The participants were offered to read the written document of how the experiment would take place. They were asked about their wellness, possible sickness, and photosensitive epilepsy. If they were sick or had any conditions that would affect the experiment results or contraindications for VR usage, they would not be allowed to participate in the experiment. Participants then signed the agreement (informed consent) for participation in the experiment.

Having done this, the participants were guided to the experimental apparatus where the experimenters helped to equip them with the HMD and explained the controls to navigate and interact in the VR game. Since the Oculus Rift S does not have the option for manual interpupillary distance (IPD) setting, it was measured manually, and Oculus Rift $S$ was configured for the optimal settings so that a mismatch would not contribute to VR Sickness.

At a time, only one participant was involved in the experiment. One researcher was conducting the experiment, while the other was observing the participants and operating the equipment. Each session lasted $90 \mathrm{~min}$ or less, including the introduction; signing the consent form; and filling out the pre-, mid-, and post-questionnaires. On average, it took approximately $18 \mathrm{~min}$ to experience the introductory tutorial/entry and all the experiment scenarios.

First, the participants played the introductory tutorial/entry level to get familiar with the VR technology (HMD and Oculus Touch controllers), virtual environment, and the game mechanics. In this entry level, participants got familiar with moving, rotating, jumping, collecting coins, and opening doors. This entry level was included in the experiment procedure so that later gameplay would be as smooth as possible. This session did not last more than $2 \mathrm{~min}$ for each participant. When equipping the HMD, the participants were instructed to close their eyes and keep them closed for a few seconds after successful placement, so that fluctuating FPS at the start of the game would not contribute to the VRISE.

The participants were seated on a comfortable sofa throughout the game (stationary VR experience). They translated and rotated their avatar (virtual body) with an Oculus Touch controller. Participants were instructed to hold both controllers in their hands, using one or both as preferred by them. Input controls were mirrored on both controllers, so moving and interactions were comfortable right-handed or left-handed. Participants could interact through the HMD by looking in any direction they wanted to explore the given scene.

After they completed the entry level, they were directed to complete the second questionnaire. They were asked about their wellness, previous usage of VR technology, physical activity level (International Physical Activity Questionnaire), and emotional state. All questionnaires at the Multimedia Laboratory were filled out online via a web browser on a notebook PC with a touch display.

Having done this, participants began to play all six levels. To account for potential order effects, conditions were counterbalanced across participants (Latin square method). Before the gameplay, the participants were not informed which level they would play (mode 
of the game), neither were they informed about the rest-frames, so they would not pay attention and be aware of the rest-frames (the instruction did not manipulate the awareness and salience of the rest-frames). Based on the post-semi-structured questionnaire, most of them noticed the glasses and only some the baseball hat. Average playtime for all three rest-frame conditions (NORF, RFG, and RFH) in HA mode of the game was 2 min and $19 \mathrm{~s}$, while, in the LA, it was 3 min and $22 \mathrm{~s}$, so the playtime in LA was $44.88 \%$ longer.

Immediately after each gameplay, participants' feedback was collected with a third set of questionnaires. We asked participants to complete the SSQ, SPES, UEQ-S, and VRNQ Questionnaire (user experience and VRISE section of the questionnaire). All those questionnaires were completed on a computer on the other side of the Multimedia Laboratory, so the participants needed to stand up and walk to the computer, assuming that would help lessen the VRISE and be more precisely able to determine them (especially postural instability and disorientation).

We also collected the FMS score six times, before and during the game. The first FMS score was collected before the participant put on the HMD, while the other FMS scores were collected during the gameplay, and the last one at the end of the gameplay, still with an HMD placed on the head. During gameplay, there were specific places in VE where FMS scores needed to be given. This was done using giant billboards in VE (Figure 4), which displayed the text "Kako se počutite?" (How do you feel?) when the player came near the billboard. FMS score was given verbally and recorded by the researcher. Before the first gameplay, the participants were instructed how to give FMS scores correctly.

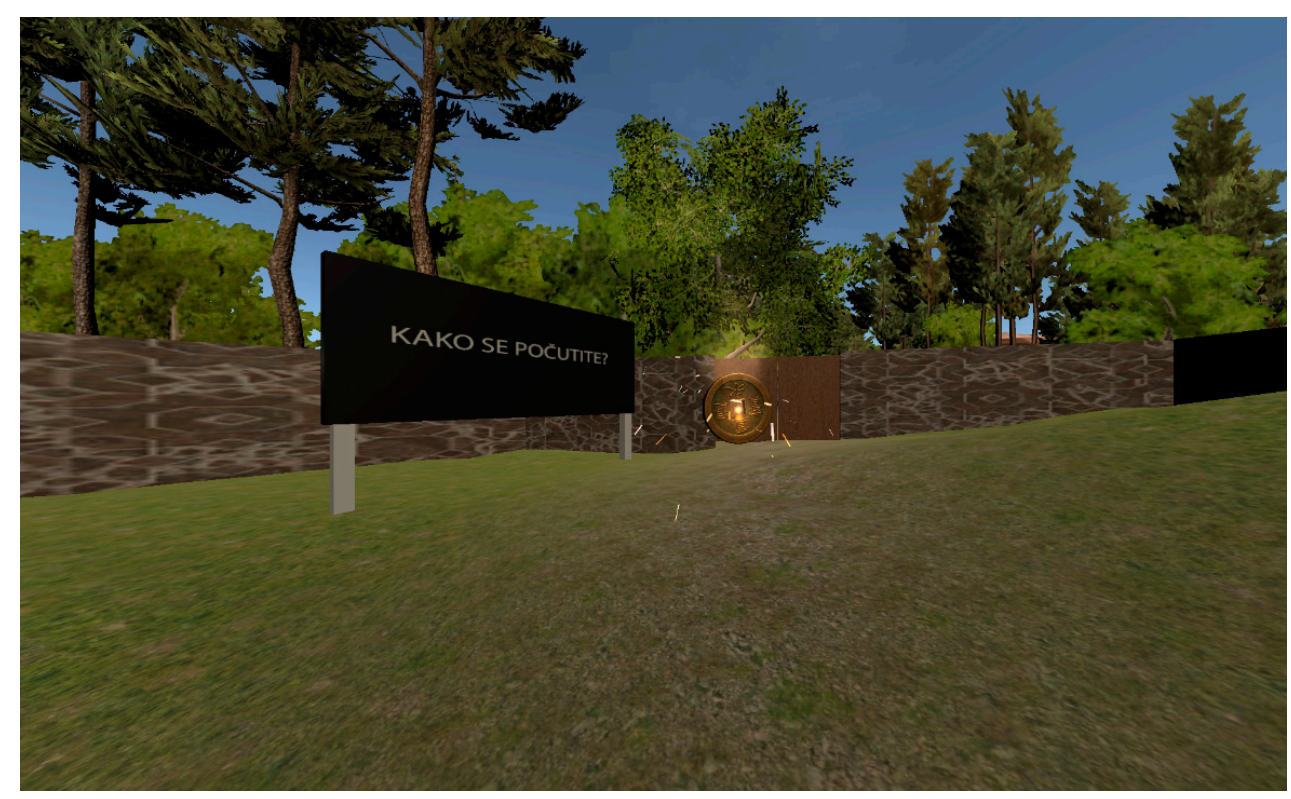

Figure 4. Screenshot from the LA game without rest-frames in front of the table for collecting the Fast Motion Sickness (FMS) score. There are visible doors in the background, which were needed to be opened to continue onto the next scene.

To continue with the next level, we gave participants enough time to recover from possible VRISE before proceeding with the next level. The minimum time to proceed with another level/condition was five minutes, but we gave them more time when needed. We took care that participants did not continue with the next level if the FMS took before gameplay was more than 1 (negligible VRISE effects).

After completing the experiment, participants were instructed to complete the fourth set of questionnaires. They were asked to fulfil a VRNQ Questionnaire (Game Mechanics and In-Game Assistance section) and NASA Task Load Index (NASA-TLX) to determine the task load of the experiment. Qualitative data was collected by a semi-structured interview 
after the experiment's competition and analyzed to investigate the potential factors causing VRISE and affecting the user experience and presence.

One week's time after the experiment, to summarize the experience with the experiment, one last questionnaire was fulfilled by the participants ( 2 of them did not complete it). We have also asked them if they experienced any side effects from VR usage, side effects that lasted for more than $24 \mathrm{~h}$, and technology acceptance questions (how probable that they will continue to use VR technology and recommend it to others, based on the experiences from the experiment).

\section{Results}

In this section, we present the results of the study. In the analysis, we took into account only the results of the participants that successfully completed all six repetitions and properly filled out the questionnaires. Eleven participants did not complete the experiment: one from lack of time, and ten of them exited the experiment due to elevated VRISE Symptoms (22.73\% Dropout Rate). According to a study [36], this is slightly above average $(15.6 \%)$ for all content types. Of those dropouts due to VR Sickness, eight were women, and two were males. Data from online questionnaires were exported into Excel, and the preparation of the data, calculations, and aggregation of the results were performed in Tableau Prep [37] and statistically analyzed in IBM SPSS [38] and R Studio [39].

Due to the directed hypothesis, planned pairwise comparisons were conducted instead of an analysis of variances. To evaluate the impacts of the rest-frames, the conditions with rest-frames (RFG and RFH) were compared to the condition without rest-frames (NORF) for both the low and high action modes of the game. Additionally, the rest-frame conditions were compared with each other. For all comparisons regarding the VRISE, user experience, presence, and performance, the significance level was set to 0.05 to conclude significant differences. Some of the developed hypotheses were directional, which permitted analyses using one-tailed statistical tests.

\subsection{VRISE}

The detailed VRISE results assessed by the SSQ, FMS, and VRNQ questionnaires for all conditions are shown in Table 2. The average FMS was calculated as an average score from all six verbally given scores before, during, and at the end for each game scenario. Progress of the average FMS scores by time and condition is presented in Figure 5.

For the LA mode of the game, it was evident that the mean SSQ scores were higher (more sickness) when rest-frames were used. Quite the opposite was apparent in the HA mode of the game, where lower mean SSQ scores (less sickness) were observed when restframes were used, except for the RFG condition on the SSQ Nausea and SSQ Oculomotor subscales, where the mean scores were higher. From the results, there was a noticeable drop in the SSQ Total and Disorientation maximum scores when comparing the HA_NORF condition to the HA_RFG and HA_RFH conditions. From those results, we can conclude that using rest-frames in the HA mode of the game influenced the maximum SSQ Total and SSQ Disorientation scores. The maximum values of the SSQ Total and SSQ Disorientation scores in the HA mode of the game with the rest-frames were even lower than in a less provocative LA mode of the game for any of the conditions.

The Shapiro-Wilk test showed that the data was not normally distributed. Therefore, we used nonparametric statistical tests. To observe the differences between the combinations of conditions for the VRISE scores and examine the hypotheses, the Wilcoxon signed-rank test was used. The results for the LA mode of the game are presented in Table 3. 


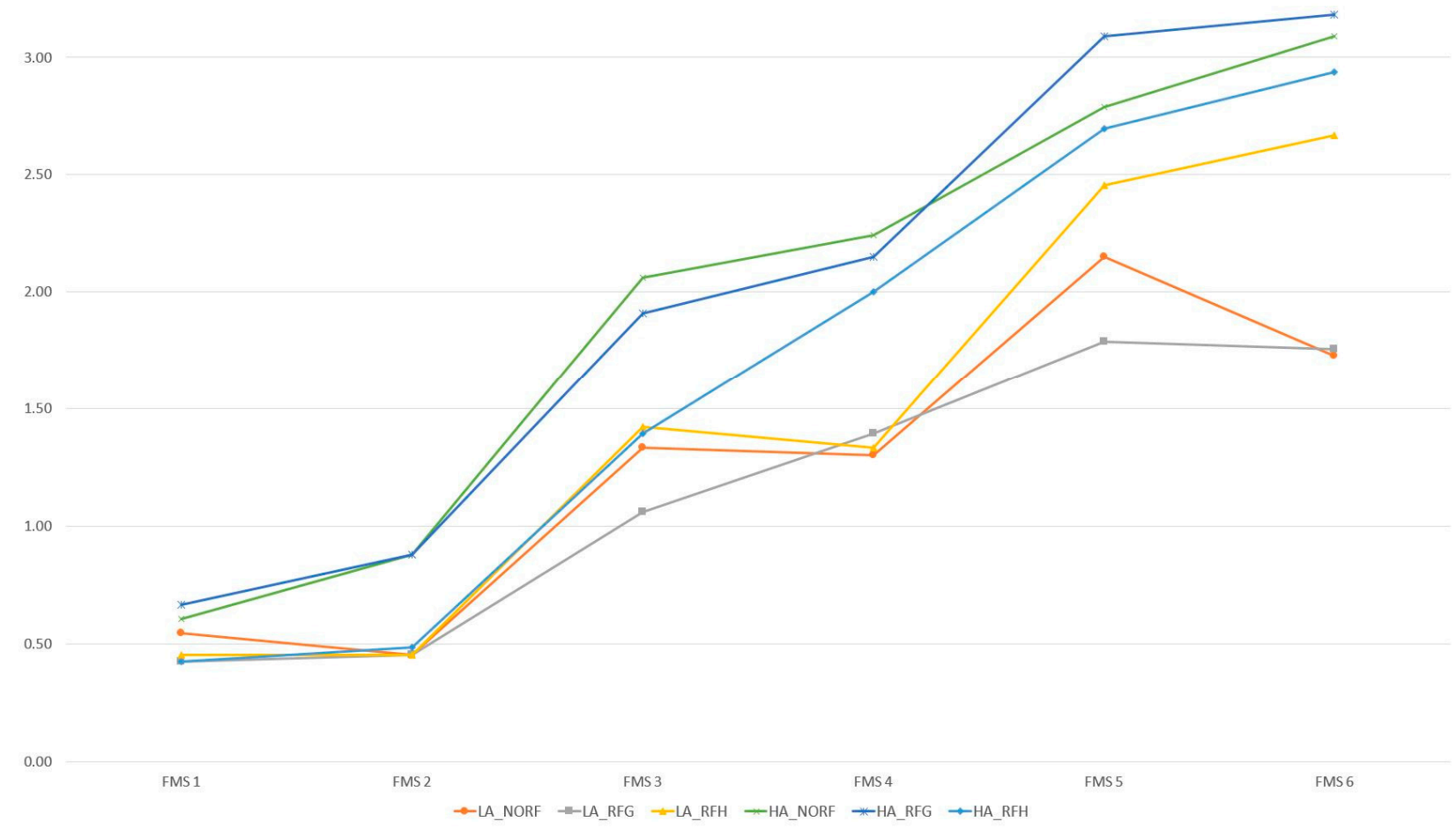

Figure 5. Progress of the average FMS scores by time and condition for all participants who completed the experiments (all six repetitions). A drop from FMS 3 to FMS 4 for the LA_NORF and LA_RFH conditions and a drop from FMS 5 to FMS 6 for the LA_NORF and LA_RFG conditions was interesting. In general, the FMS scores rose throughout the gameplay for all the conditions. The condition without the rest-frames is referred to as NORF, condition with rest-frames glasses as RFG, and condition with the baseball hat as RFH.

Table 2. Subjective Virtual Reality-Induced Symptoms and Effects (VRISE) levels assessed by the Simulator Sickness Questionnaire (SSQ), Fast Motion Sickness (FMS), and Virtual Reality Neuroscience Questionnaire (VRNQ) (VRISE Section).

\begin{tabular}{|c|c|c|c|c|c|c|}
\hline VRISE Scale & Condition & $\mathbf{N}$ & Mean & SD & Min Value & Max Value \\
\hline \multirow[t]{6}{*}{ SSQ Total } & LA_NORF & 33 & 21.78 & 23.04 & 0 & 104.72 \\
\hline & LA_RFG & 33 & 27.31 & 26.70 & 0 & 119.68 \\
\hline & LA_RFH & 33 & 30.49 & 30.49 & 0 & 123.42 \\
\hline & HA_NORF & 33 & 27.88 & 27.75 & 0 & 112.20 \\
\hline & HA_RFG & 33 & 27.77 & 25.11 & 0 & 78.54 \\
\hline & HA_RFH & 33 & 25.84 & 22.74 & 0 & 71.06 \\
\hline SSQ & LA_NORF & 33 & 21.93 & 29.33 & 0 & 139.20 \\
\hline \multirow[t]{5}{*}{ Disorientation } & LA_RFG & 33 & 31.21 & 35.32 & 0 & 125.28 \\
\hline & LA_RFH & 33 & 32.90 & 36.96 & 0 & 167.04 \\
\hline & HA_NORF & 33 & 31.64 & 34.15 & 0 & 125.28 \\
\hline & HĀ_RFG & 33 & 27.42 & 28.16 & 0 & 83.52 \\
\hline & HA_RFH & 33 & 27.84 & 27.18 & 0 & 83.52 \\
\hline \multirow[t]{6}{*}{ SSQ Nausea } & LA_NORF & 33 & 23.99 & 24.45 & 0 & 95.40 \\
\hline & LA_RFG & 33 & 26.60 & 26.38 & 0 & 95.40 \\
\hline & LA_RFH & 33 & 32.96 & 35.38 & 0 & 114.48 \\
\hline & HA_NORF & 33 & 30.35 & 34.76 & 0 & 124.02 \\
\hline & HA_RFG & 33 & 31.22 & 33.58 & 0 & 114.48 \\
\hline & HA_RFH & 33 & 28.04 & 30.35 & 0 & 95.40 \\
\hline SSQ & LA_NORF & 33 & 13.32 & 17.98 & 0 & 90.96 \\
\hline \multirow[t]{5}{*}{ Oculomotor } & LĀ_RFG & 33 & 17.23 & 19.81 & 0 & 98.54 \\
\hline & LA_RFH & 33 & 17.69 & 19.11 & 0 & 90.96 \\
\hline & HA_NORF & 33 & 15.16 & 16.19 & 0 & 68.22 \\
\hline & HĀ_RFG & 33 & 16.54 & 16.78 & 0 & 68.22 \\
\hline & HA_RFH & 33 & 14.93 & 17.00 & 0 & 75.80 \\
\hline
\end{tabular}


Table 2. Cont.

\begin{tabular}{ccccccc}
\hline VRISE Scale & Condition & N & Mean & SD & Min Value & Max Value \\
\hline FMS & LA_NORF & 33 & 1.25 & 1.81 & 0 & 7.50 \\
Average & LA_RFG & 33 & 1.15 & 1.52 & 0 & 6.17 \\
& LA_RFH & 33 & 1.46 & 1.86 & 0 & 6.17 \\
& HA_NORF & 33 & 1.94 & 2.76 & 0 & 11.00 \\
& HA_RFG & 33 & 1.98 & 2.71 & 0 & 11.67 \\
& HA_RFH & 33 & 1.66 & 2.03 & 0 & 7.33 \\
\hline VRISE scale & LA_NORF & 33 & 32.00 & 3.73 & 20 & 35 \\
& LA_RFG & 32 & 31.28 & 4.42 & 15 & 35 \\
& LA_RFH & 33 & 31.97 & 3.32 & 21 & 35 \\
& HA_NORF & 33 & 30.97 & 4.34 & 20 & 35 \\
& HA_RFG & 33 & 31.03 & 4.57 & 19 & 35 \\
\end{tabular}

Table 3. Wilcoxon signed-rank test for the SSQ scores between the no rest-frame and restframes conditions.

\begin{tabular}{cccc}
\hline VRISE Scale & Condition & Z & $p$ \\
\hline SSQ Total & LA_NORF-LA_RFG & $-\mathbf{1 . 7 4 7}$ & $\mathbf{0 . 0 4 1}$ \\
& LA_NORF-LA_RFH & $-\mathbf{2 . 3 9 6}$ & $\mathbf{0 . 0 0 8}{ }^{*}$ \\
\hline SSQ & LA_NORF-LA_RFG & $\mathbf{- 2 . 0 1 2}$ & $\mathbf{0 . 0 2 2}{ }^{*}$ \\
Disorientation & LA_NORF-LA_RFH & $\mathbf{- 2 . 2 9 2}$ & $\mathbf{0 . 0 1 0}^{*}$ \\
\hline SSQ Nausea & LA_NORF-LA_RFG & -0.920 & 0.185 \\
& LA_NORF-LA_RFH & $-\mathbf{2 . 2 2 2}$ & $\mathbf{0 . 0 1 2}$ \\
& HA_NORF-HA_RFG & -0.190 & 0.431 \\
& HA_NORF-HA_RFH & -0.062 & 0.479 \\
\hline Oculomotor & LA_NORF-LA_RFG & $-\mathbf{1 . 6 9 3}$ & $\mathbf{0 . 0 4 7}{ }^{*}$ \\
& LA_NORF-LA_RFH & $\mathbf{- 1 . 9 5 1}$ & $\mathbf{0 . 0 2 8}$ \\
& HA_NORF-HA_RFG & -0.836 & 0.209 \\
& HA_NORF-HA_RFH & -0.489 & 0.318 \\
\hline
\end{tabular}

${ }^{*}$ and bold $=p<0.05$ (1-tailed significance).

For the SSQ scores, the one-tailed Wilcoxon signed-rank test showed statistically significant differences in the LA mode of the game for the SSQ Total and all SSQ subscales when comparing the no rest-frame to the rest-frame conditions, except for the LA_NORFLA_RFG combination for the SSQ Nausea subscale.

No statistically significant differences were observed for the HA mode of the game, and also, no statistically significant differences were observed for the FMS and VRNQVRISE subscale scores. This did not support hypothesis H1, since using rest-frames in the LA mode of the game did have an opposite (negative) effect on the SSQ scores (more sickness). Additionally, no significant differences between the rest-frame conditions were observed for both modes of the game for all VRISE scales, which did not support hypothesis $\mathrm{H} 2$ in terms of the VRISE levels.

Comparing the modes of the game, the comparisons presented in Table 4 are only made between the conditions without rest-frames, since, as it is noticeable from Table 2, there is an opposite effect of rest-frames in the LA and HA modes of the game, although, for HA, the difference is not statistically significant. Hypothesis H6 was supported for the SSQ Disorientation subscale and the FMS Average scale, since statistically significant differences were observed only between conditions LA_NORF and HA_NORF of the SSQ Disorientation subscale and LA_NORF and HA_NORF of FMS Average scale. The subjects reported significantly fewer disorientation symptoms and significantly fewer sickness symptoms assessed by the FMS Questionnaire when playing the LA mode of the game.

\subsection{User Experience}

Detailed user experience results assessed by the UEQ-S and VRNQ questionnaire are shown in Table 5. For both modes of the game, it is evident that the mean UEQ-S user 
experience scores were higher (better user experience) when rest-frames were not used, with the exception of the UEQ-S Hedonic quality scale in the LA mode of the game, and VRNQUser experience subscale in the HA mode of the game for both rest-frame conditions.

Table 4. Wilcoxon signed-rank test for the VRISE scores between mode of the game (LA vs. HA).

\begin{tabular}{|c|c|c|c|}
\hline VRISE Scale & Condition & $\mathbf{Z}$ & $p$ \\
\hline SSQ Total & $\begin{array}{l}\text { LA_NORF- } \\
\text { HA_NORF }\end{array}$ & -1.300 & 0.099 \\
\hline SSQ Disorientation & $\begin{array}{l}\text { LA_NORF- } \\
\text { HA_NORF }\end{array}$ & -1.889 & 0.030 * \\
\hline SSQ Nausea & $\begin{array}{l}\text { LA_NORF- } \\
\text { HA_NORF }\end{array}$ & -0.948 & 0.181 \\
\hline SSQ Oculomotor & $\begin{array}{l}\text { LA_NORF- } \\
\text { HA_NORF }\end{array}$ & -0.714 & -0.248 \\
\hline FMS Average & $\begin{array}{l}\text { LA_NORF- } \\
\text { HA_NORF }\end{array}$ & -2.103 & 0.017 * \\
\hline $\begin{array}{l}\text { VRNQ-VRISE } \\
\text { subscale }\end{array}$ & $\begin{array}{l}\text { LA_NORF- } \\
\text { HA_NORF }\end{array}$ & -1.322 & 0.098 \\
\hline
\end{tabular}

${ }^{*}$ and bold $=p<0.05$ (1-tailed significance).

Table 5. Subjective user experience levels assessed by the short version of the User Experience Questionnaire (UEQ-S) and VRNQ (User Experience Section) Questionnaire.

\begin{tabular}{|c|c|c|c|c|c|c|}
\hline User Experience Scale & Condition & $\mathbf{N}$ & Mean & SD & Min Value & Max Value \\
\hline UEQ-S & LA_NORF & 33 & 1.37 & 0.96 & -1.25 & 3.00 \\
\hline \multirow{5}{*}{ Overall } & LA_RFG & 33 & 1.13 & 1.18 & -1.88 & 3.00 \\
\hline & LA_RFH & 33 & 1.30 & 1.02 & -1.25 & 3.00 \\
\hline & HA_NORF & 33 & 1.62 & 0.74 & 0.00 & 3.00 \\
\hline & HA_RFG & 33 & 1.53 & 0.92 & -0.13 & 3.00 \\
\hline & HA_RFH & 33 & 1.39 & 1.00 & -1.00 & 3.00 \\
\hline UEQ-S & LA_NORF & 33 & 2.03 & 0.77 & 0.00 & 3.00 \\
\hline Pragmatic & LA_RFG & 33 & 1.48 & 1.15 & -1.50 & 3.00 \\
\hline \multirow[t]{4}{*}{ Quality } & LA_RFH & 33 & 1.69 & 0.95 & -0.25 & 3.00 \\
\hline & HA_NORF & 33 & 1.82 & 0.84 & -0.25 & 3.00 \\
\hline & HA_RFG & 33 & 1.79 & 0.97 & -0.25 & 3.00 \\
\hline & HA_RFH & 33 & 1.71 & 0.97 & 0.00 & 3.00 \\
\hline UEQ-S & LA_NORF & 33 & 0.70 & 1.58 & -3.00 & 3.00 \\
\hline Hedonic & LĀ_RFG & 33 & 0.79 & 1.57 & -2.50 & 3.00 \\
\hline \multirow[t]{4}{*}{ Quality } & LA_RFH & 33 & 0.92 & 1.47 & -2.75 & 3.00 \\
\hline & HA_NORF & 33 & 1.43 & 1.06 & -0.75 & 3.00 \\
\hline & HA_RFG & 33 & 1.27 & 1.28 & -2.00 & 3.00 \\
\hline & HA_RFH & 33 & 1.08 & 1.4 & -2.50 & 3.00 \\
\hline VRNQ & LA_NORF & 32 & 23.31 & 3.98 & 18.00 & 33.00 \\
\hline User & LĀ_RFG & 31 & 23.00 & 3.86 & 16.00 & 30.00 \\
\hline Experience & LA_RFH & 33 & 22.76 & 4.06 & 13.00 & 31.00 \\
\hline \multirow[t]{3}{*}{ scale } & HA_NORF & 32 & 23.59 & 4.62 & 14.00 & 33.00 \\
\hline & HA_RFG & 30 & 24.37 & 4.57 & 18.00 & 33.00 \\
\hline & HA_RFH & 33 & 24.12 & 4.52 & 14.00 & 33.00 \\
\hline
\end{tabular}

The Shapiro-Wilk test showed that the data was not normally distributed for all variables, so we used nonparametric statistical tests. To observe the differences between the combinations of conditions for the user experience scores and examine the hypotheses, the Wilcoxon signed-rank test was used as presented in Table 6.

For the user experience scores, the resulting two-tailed Wilcoxon signed-rank test showed only one statistically significant difference in the LA mode of the game for the UEQ-S Pragmatic Quality between the LA_NORF and LA_RFG conditions. This partially supported hypothesis $\mathrm{H} 3$ that rest-frames would not affect the user experience, except in the LA mode of the game, where the user experience scores for UEQ-S Pragmatic Quality were significantly lower when rest-frame glasses were used. 
Table 6. Wilcoxon signed-rank test for user experience scores assessed by the UEQ-S Questionnaire between the no rest-frame and rest-frames conditions.

\begin{tabular}{cccc}
\hline User Experience Scale & Condition & Z & $p$ \\
\hline UEQ-S & LA_NORF-LA_RFG & -1.701 & 0.090 \\
Overall & LA_NORF-LA_RFH & -0.891 & 0.382 \\
& HA_NORF-HA_RFG & -0.932 & 0.360 \\
& HA_NORF- & -1.594 & 0.113 \\
\hline HEQ-S & HA_RFH & $-\mathbf{2 . 8 6 6}$ & $\mathbf{0 . 0 0 3}{ }^{*}$ \\
Pragmatic & LA_NORF-LA_RFG & -1.883 & 0.059 \\
Quality & HA_NORF-HA_RFG & -0.180 & 0.872 \\
& HA_NORF- & -1.122 & 0.272 \\
& HA_RFH & -0.017 & 0.993 \\
UEQ-S & LA_NORF-LA_RFG & -0.503 & 0.626 \\
Hedonic & LA_NORF-LA_RFH & -1.605 & 0.115 \\
& HA_NORF-HA_RFG & -1.907 & 0.056 \\
\hline & HA_NORF- & & \\
\hline
\end{tabular}

*and bold $=p<0.05$ (2-tailed significance).

No statistically significant differences were observed for the HA mode of the game, and also, no statistically significant differences were observed for the VRNQ-User Experience scores. Additionally, no significant differences between the rest-frame conditions were observed for both modes of the game for all user experience scales, which did not support hypothesis $\mathrm{H} 2$ in terms of user experience levels.

Comparing modes of the game, comparisons are presented in Table 7. Hypothesis H7 was supported for the UEQ-S Overall scores for the RFG condition, for the UEQ-S Hedonic Quality scores for the NORF and the RFG conditions, and the VRNQ-User Experience scores for all conditions. Hypothesis H7 was not supported for the UEQ-S Pragmatic Quality subscale data, where the UEQ-S Pragmatic Quality scores were significantly lower in the HA mode of the game for the NORF condition.

Table 7. Wilcoxon signed-rank test for user experience scores assessed by the UEQ-S and VRNQ (User Experience Section) Questionnaire between the modes of the game (LA vs. HA).

\begin{tabular}{|c|c|c|c|}
\hline UX Scale & Condition & $\mathbf{Z}$ & $p$ \\
\hline UEQ-S & $\begin{array}{l}\text { LA_NORF- } \\
\text { HA_NORF }\end{array}$ & -1.439 & 0.078 \\
\hline Overall & $\begin{array}{l}\text { LA_RFG-HA_RFG } \\
\text { LA_RFH-HA_RFH }\end{array}$ & $\begin{array}{l}-2.713 \\
-0.589\end{array}$ & $\begin{array}{c}\mathbf{0 . 0 0 3} * \\
0.284\end{array}$ \\
\hline UEQ-S & $\begin{array}{l}\text { LA_NORF- } \\
\text { HA_NORF }\end{array}$ & -1.846 & 0.034 * \\
\hline Pragmatic & LA_RFG-HA_RFG & -1.588 & 0.058 \\
\hline Quality & LA_RFH-HA_RFH & -0.314 & 0.382 \\
\hline UEQ-S & $\begin{array}{l}\text { LA_NORF- } \\
\text { HA_NORF }\end{array}$ & -3.836 & $0.000 *$ \\
\hline Hedonic & LA_RFG-HA_RFG & -2.839 & $0.002 *$ \\
\hline Quality & LA_RFH-HA_RFH & -1.167 & 0.125 \\
\hline VRNQ & $\begin{array}{l}\text { LA_NORF- } \\
\text { HA_NORF }\end{array}$ & -1.733 & $0.042 *$ \\
\hline User Experience & LA_RFG-HA_RFG & -2.680 & $0.003 *$ \\
\hline subscale & LA_RFH-HA_RFH & -2.836 & $0.002 *$ \\
\hline
\end{tabular}

*and bold $=p<0.05$ (1-tailed significance).

\subsection{Presence}

The detailed presence results assessed by the SPES questionnaire for all conditions are shown in Table 8. 
Table 8. Subjective presence levels assessed by the Spatial Presence Experience Scale (SPES) Questionnaire.

\begin{tabular}{ccccccc}
\hline SPES Scale & Condition & N & Mean & SD & Min Value & Max Value \\
\hline SPES Total & LA_NORF & 33 & 27.24 & 6.796 & 9 & 40 \\
& LA_RFG & 33 & 26.00 & 7.018 & 8 & 39 \\
& LA_RFH & 33 & 26.67 & 6.542 & 13 & 39 \\
& HA_NORF & 33 & 27.61 & 6.339 & 11 & 40 \\
& HA_RFG & 33 & 27.94 & 6.614 & 10 & 40 \\
& HA_RFH & 33 & 27.67 & 6.836 & 8 & 40 \\
\hline SPES & LA_NORF & 33 & 13.24 & 4.366 & 4 & 20 \\
Self-location & LA_RFG & 33 & 12.55 & 4.487 & 4 & 19 \\
& LA_RFH & 33 & 13.03 & 4.073 & 4 & 19 \\
& HA_NORF & 33 & 13.39 & 3.864 & 4 & 20 \\
& HA_RFG & 33 & 13.48 & 4.1 .09 & 5 & 20 \\
SPES & HA_RFH & 33 & 13.61 & 3.921 & 4 & 20 \\
Possible & LA_NORF & 33 & 14.00 & 3.192 & 5 & 20 \\
Actions & LA_RFG & 33 & 13.45 & 3.270 & 4 & 20 \\
& LA_RFH & 33 & 13.64 & 3.239 & 5 & 20 \\
& HA_NORF & 33 & 14.21 & 3.267 & 7 & 20 \\
& HA_RFG & 33 & 14.45 & 3.401 & 5 & 20 \\
& HA_RFH & 33 & 14.06 & 3.665 & 4 & 20 \\
\hline
\end{tabular}

For the LA mode of the game, it is evident that the mean SPES scores were lower (worsened presence) when the rest-frames were used, and for the HA mode of the game, it is evident that the SPES scores were higher when the rest-frames were used, with the exception of the SPES Possible Actions score in the HA_RFH condition. Those mixed results can be related to the elevated VRISE scores in the LA mode the game.

The Shapiro-Wilk test showed that the data was not normally distributed for all variables, so we used nonparametric statistical tests. To observe the differences between the combinations of conditions for the SPES scores and examine the hypothesis, the Wilcoxon signed-rank test was used as presented in Table 9.

Table 9. Wilcoxon signed-rank test for presence-SPES scores between the no rest-frame and restframes conditions.

\begin{tabular}{cccc}
\hline SPES Scale & Condition & Z & $p$ \\
\hline SPES Total & LA_NORF-LA_RFG & $-\mathbf{2 . 2 8 3}$ & $\mathbf{0 . 0 1 0}$ \\
& LA_NORF-LA_RFH & -1.165 & 0.128 \\
& HA_NORF-HA_RFG & -0.871 & 0.189 \\
& HA_NORF-HA_RFH & -0.539 & 0.304 \\
\hline Self-location & LA_NORF-LA_RFG & $\mathbf{- 1 . 9 8 8}$ & $\mathbf{0 . 0 2 4}$ \\
& LA_NORF-LA_RFH & -0.385 & 0.359 \\
& HA_NORF-HA_RFG & -0.300 & 0.394 \\
Possible Actions & HA_NORF-HA_RFH & -1.174 & 0.129 \\
\hline SPES & LA_NORF-LA_RFG & $\mathbf{- 1 . 8 8 5}$ & $\mathbf{0 . 0 3 0}$ \\
& LA_NORF-LA_RFH & -0.911 & 0.187 \\
& HA_NORF-HA_RFG & -0.531 & 0.309 \\
& HA_NORF-HA_RFH & -0.038 & 0.490 \\
\hline
\end{tabular}

${ }^{*}$ and bold $=p<0.05$ (1-tailed significance).

We assumed that using rest-frames would positively affect the presence. Still, we found a statistically significant difference between the LA_NORF and LA_RFG conditions for the SPES Total score and the SPES Self-location and SPES Possible actions subscales. Using rest-frame glasses in the LA mode of the game did affect the presence negatively (less presence), which did not support hypothesis H4. This might be related to the elevated VRISE levels in the LA mode of the game when rest-frames were used. However, the effect 
size was very small. The presence was not affected when the baseball hat was used as a rest-frame in the LA mode of the game.

No statistically significant differences were observed for the HA mode of the game. Although our hypothesis $\mathrm{H} 4$ was not confirmed, we found those results favorable, since the presence was mostly not affected by rest-frames usage. Additionally, no significant differences between the rest-frame conditions were observed for both modes of the game for all SPES scales, which did not support hypothesis $\mathrm{H} 2$ in terms of the presence levels.

Based on cognitive involvement, a factor of spatial presence, spatial presence should be higher in HA levels of the game. When users are preoccupied with media stimuli and are highly involved with media content, they are cognitively involved. Their mental capacity is primarily devoted to the media and not to reality [30]. In our game, players were more cognitively involved while playing the high action game due to moving faster and rotating, jumping, and more coins that needed to be collected. Interestingly, we could confirm the impact of cognitive involvement on presence only for the RFG conditions of the game for the SPES Total scores, as well as for the SPES Self-location and SPES Possible Actions subscales, as shown in Table 10, which supported hypothesis H8.

Table 10. Wilcoxon signed-rank test for presence-SPES scores between the modes of the game (LA vs. HA).

\begin{tabular}{cccc}
\hline SPES Scale & Condition & Z & $p$ \\
\hline SPES Total & LA_NORF-HA_NORF & -0.845 & 0.205 \\
& LA_RFG-HA_RFG & $-\mathbf{2 . 6 9 9}$ & $\mathbf{0 . 0 0 3}$ \\
& LA_RFH-HA_RFH & -1.512 & 0.067 \\
\hline SPES & LA_NORF-HA_NORF & -0.702 & 0.249 \\
Self-location & LA_RFG-HA_RFG & $-\mathbf{2 . 2 0 5}$ & $\mathbf{0 . 0 1 4}$ \\
& LA_RFH-HA_RFH & -1.406 & 0.083 \\
\hline SPES & LA_NORF-HA_NORF & -0.530 & 0.301 \\
Possible Actions & LA_RFG-HA_RFG & $-\mathbf{2 . 4 0 3}$ & $\mathbf{0 . 0 0 7}$ \\
& LA_RFH-HA_RFH & -0.798 & 0.215 \\
\hline
\end{tabular}

*and bold $=p<0.05$ (1-tailed significance).

\subsection{Performance (Score and Time)}

We also recorded the performances in the gameplay of users. Scores obtained by collecting coins and completion time in seconds for each level completed were collected. Detailed results of the score and competition time in seconds for all conditions are shown in Table 11 and presented in Figures 6 and 7. The maximum possible achieved score was 55 in the LA mode of the game and 79 in the HA mode of the game (if the participants would collect all the coins).

Table 11. Performance-score and time per condition.

\begin{tabular}{ccccccc}
\hline & Condition & N & Mean & SD & Min Value & Max Value \\
\hline Time (s) & LA_NORF & 32 & 203.53 & 35.88 & 166 & 300 \\
& LA_RFG & 33 & 208.36 & 42.86 & 166 & 357 \\
& LA_RFH & 32 & 206.84 & 29.31 & 168 & 281 \\
& HA_NORF & 32 & 138.09 & 33.86 & 82 & 229 \\
& HA_RFG & 33 & 139.76 & 35.87 & 82 & 250 \\
& HA_RFH & 33 & 144.82 & 40.11 & 87 & 257 \\
\hline Score & LA_NORF & 33 & 54.58 & 0.83 & 51 & 55 \\
& LA_RFG & 33 & 54.70 & 0.81 & 51 & 55 \\
& LA_RFH & 32 & 54.50 & 0.67 & 53 & 55 \\
& HA_NORF & 32 & 69.47 & 5.64 & 56 & 79 \\
& HA_RFG & 33 & 68.94 & 5.49 & 56 & 77 \\
& HA_RFH & 33 & 69.55 & 6.56 & 56 & 79 \\
\hline
\end{tabular}




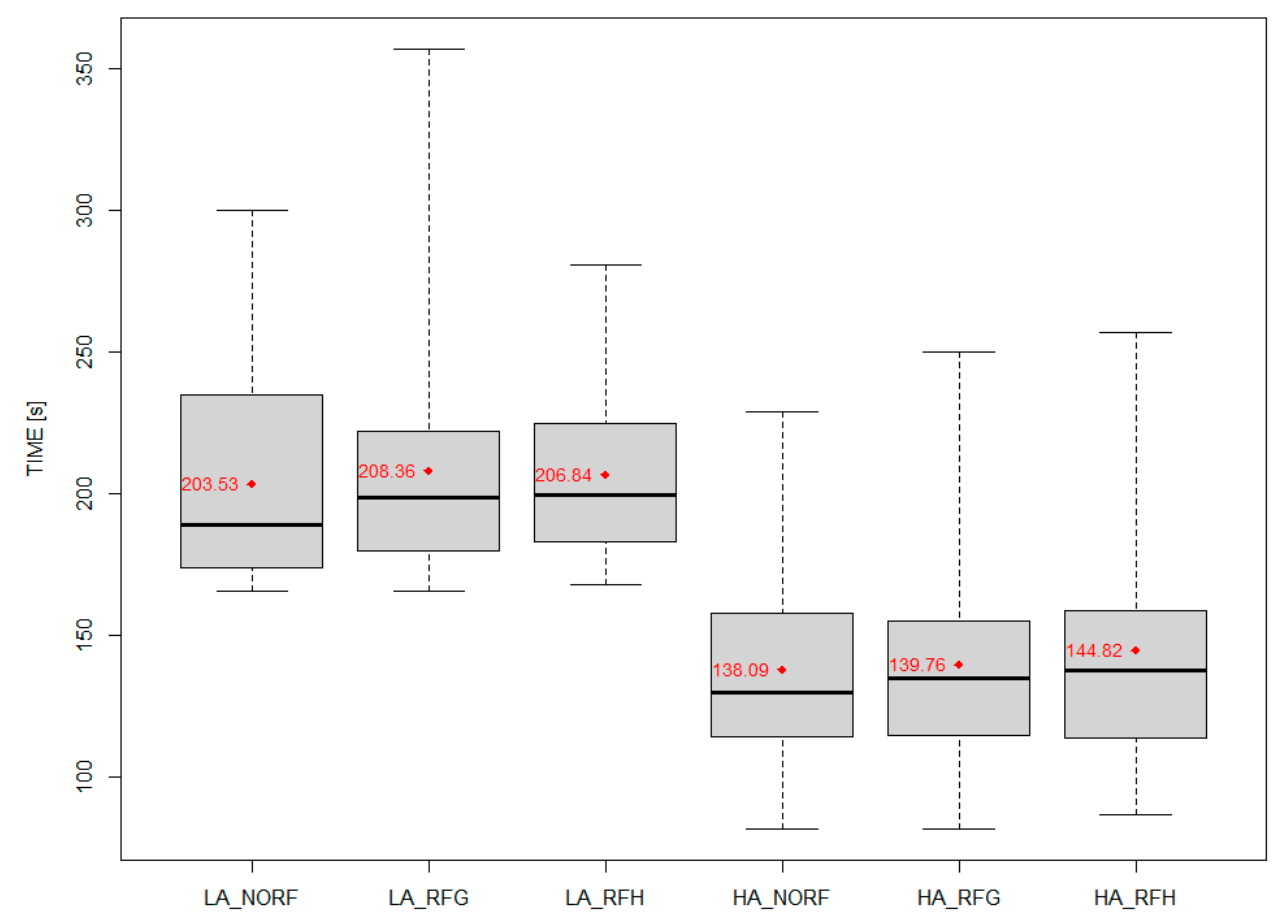

Figure 6. Boxplots of the completion times for all conditions (with red circles marking and labeling the mean values of the completion times).

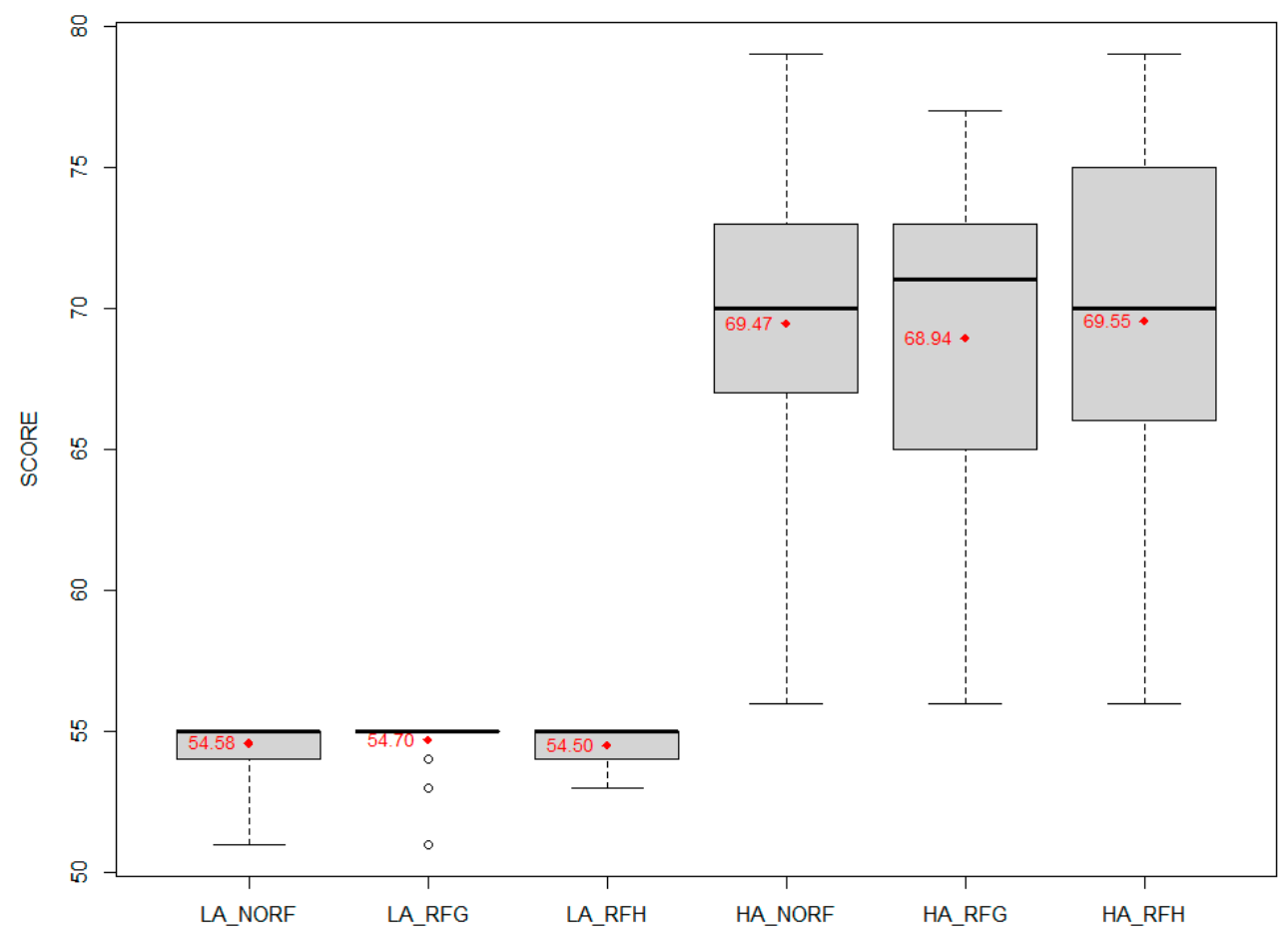

Figure 7. Boxplots of achieved scores for all conditions (with red circles marking and labeling the mean values of the scores).

For both the LA and HA modes of the game, it is evident that the mean completion times are higher when rest-frames were used.

The Shapiro-Wilk test showed that the data was not normally distributed for all variables, so we used nonparametric statistical tests. To observe the differences between the combinations of all conditions for the performance scores and examine the hypotheses, the Wilcoxon signed-rank test was used as presented in Table 12. 
Table 12. Wilcoxon signed-rank test for the performance (score and time) scores between the restframes conditions.

\begin{tabular}{cccc}
\hline & Condition & Z & $p$ \\
\hline \multirow{2}{*}{ Time } & LA_NORF-LA_RFG & -0.862 & 0.396 \\
& LA_NORF-LA_RFH & -0.094 & 0.930 \\
& HA_NORF-HA_RFG & -0.137 & 0.896 \\
& HA_NORF-HA_RFH & -0.980 & 0.334 \\
\hline \multirow{2}{*}{ Score } & LA_NORF-LA_RFG & -0.811 & 0.520 \\
& LA_NORF-LA_RFH & -0.355 & 0.842 \\
& HA_NORF-HA_RFG & -0.697 & 0.495 \\
& HA_NORF-HA_RFH & -0.157 & 0.880 \\
\hline
\end{tabular}

We did not find any statistically significant differences between the no rest-frame and rest-frame conditions, so we can conclude that the usage of head-centric rest-frames did not affect the performance. Those findings supported our Hypothesis H5. Additionally, no significant differences between the rest-frame conditions were observed for both modes of the game for performance scores, which did not support Hypothesis H2 in terms of the performance levels.

\subsection{Impact of Previous Experience with VR Technology}

We confirmed that the usage of head-centric rest-frames did elevate the VRISE levels in the LA mode of the game for both types of rest-frames. However, examining the results of the VRISE levels in the HA mode of the game, it was noticeable that the mean SSQ Total score and the SSQ Disorientation score were lower, and also, the maximum SSQ Total and Disorientation scores were noticeably lower when rest-frames were used. Those differences were not statistically significant, so a conclusion was not possible.

Therefore, we examined different profiles of the participants based on education, gender, age, body mass index (BMI), gaming experience, vision problems, dominant eye, physical activity level, headaches history, susceptibility to motion sickness, ITQ, BFI, IPAQ, etc. to find any significant differences for the VRISE scores in the HA mode of the game. We found a subgroup of participants who had significantly lower SSQ Disorientation scores in the HA mode of the game when rest-frames glasses were used. Those were the participants who had previous experience with VR Technology.

Detailed SSQ results of the participants with previous experience with VR technology, assessed by the SSQ questionnaire for all conditions, are shown in Table 13.

For the LA mode of the game, it was evident that the mean SSQ scores (more sickness) were higher when the rest-frames were used compared to the no rest-frame condition, except for condition LA_RFG on the SSQ Nausea scale. Quite the opposite was evident in the HA mode of the game, where lower mean SSQ scores (less sickness) were observed when rest-frames were used, except for the LA_RFG condition for the SSQ Nausea subscale, where the mean scores were higher. From the results, there was a noticeable drop in the SSQ Total and Disorientation maximum scores when comparing the HA_NORF condition to the HA_RFG and HA_RFH conditions. From those results, we can conclude that the usage of rest-frames in the HA mode of the game influenced the maximum SSQ total and SSQ disorientation scores.

The Shapiro-Wilk test showed that the data was not normally distributed, so we used nonparametric statistical tests. To observe the differences between the combinations of conditions for the SSQ scores and examine the hypotheses, the Wilcoxon signed-rank test was used as presented in Table 14.

For the SSQ scores, the resulting one-tailed Wilcoxon signed-rank test showed a statistically significant difference in the HA mode of the game for the HA_NORF-HA_RFG combination for the SSQ Disorientation scores. However, the HA_NORF and HA_RFH combinations approached statistical significance for the SSQ Disorientation scores. This supported hypothesis H1 for the SSQ Disorientation subscale, since using rest-frame 
glasses in the HA mode of the game significantly lowered the SSQ Disorientation scores (less disorientation-related side effects) for users experienced with VR Technology.

Table 13. Subjective VRISE levels assessed by the SSQ, FMS, and VRNQ (VRISE Section) Questionnaire for the participants experienced with VR Technology.

\begin{tabular}{ccccccc}
\hline VRISE Scale & Condition & N & Mean & SD & Min Value & Max Value \\
\hline SSQ Total & LA_NORF & 17 & 23.76 & 19.88 & 0.00 & 63.58 \\
& LA_RFG & 17 & 24.42 & 18.94 & 3.74 & 71.06 \\
& LA_RFH & 17 & 31.90 & 27.77 & 0.00 & 93.50 \\
& HA_NORF & 17 & 34.76 & 33.58 & 0.00 & 112.20 \\
& HA_RFG & 17 & 32.12 & 23.62 & 3.74 & 78.54 \\
& HA_RFH & 17 & 27.28 & 23.42 & 0.00 & 67.32 \\
\hline SSQ & LA_NORF & 17 & 23.75 & 24.98 & 0.00 & 83.52 \\
& LA_RFG & 17 & 30.30 & 32.36 & 0.00 & 111.36 \\
& LA_RFH & 17 & 33.57 & 30.36 & 0.00 & 97.44 \\
& HA_NORF & 17 & 42.57 & 39.21 & 0.00 & 125.28 \\
& HA_RFG & 17 & 31.93 & 28.60 & 0.00 & 83.52 \\
SSQ Nausea & HA_RFH & 17 & 30.30 & 27.06 & 0.00 & 83.52 \\
& LA_NORF & 17 & 27.49 & 26.96 & 0.00 & 95.40 \\
& LA_RFG & 17 & 23.57 & 19.99 & 0.00 & 57.24 \\
& LA_RFH & 17 & 35.35 & 34.52 & 0.00 & 104.94 \\
& HA_NORF & 17 & 36.48 & 40.93 & 0.00 & 124.02 \\
& HA_RFG & 17 & 37.60 & 33.30 & 0.00 & 114.48 \\
& HA_RFH & 17 & 31.99 & 34.55 & 0.00 & 95.40 \\
\hline SSQ & LA_NORF & 17 & 13.38 & 12.72 & 0.00 & 45.48 \\
& LA_RFG & 17 & 14.27 & 11.33 & 7.58 & 53.06 \\
& LA_RFH & 17 & 18.28 & 16.31 & 0.00 & 53.06 \\
& HA_NORF & 17 & 18.28 & 18.58 & 0.00 & 68.22 \\
& HA_RFG & 17 & 17.84 & 11.97 & 0.00 & 37.90 \\
& HA_RFH & 17 & 13.38 & 12.14 & 0.00 & 37.90 \\
\hline
\end{tabular}

Table 14. Wilcoxon signed-rank test for the SSQ scores between the no rest-frame and rest-frames conditions for users experienced with VR technology.

\begin{tabular}{cccc}
\hline VRISE Scale & Condition & Z & $p$ \\
\hline SSQ Total & LA_NORF-LA_RFG & -0.542 & 0.306 \\
& LA_NORF-LA_RFH & -1.452 & 0.079 \\
& HA_NORF-HA_RFG & -0.535 & 0.307 \\
& HA_NORF-HA_RFH & -1.140 & 0.136 \\
\hline SSQ & LA_NORF-LA_RFG & -1.184 & 0.126 \\
Disorientation & LA_NORF-LA_RFH & -1.273 & 0.112 \\
& HA_NORF-HA_RFG & $-\mathbf{1 . 7 9 6}$ & $\mathbf{0 . 0 3 5}$ \\
& HA_NORF-HA_RFH & -1.704 & 0.055 \\
\hline SSQ Nausea & LA_NORF-LA_RFG & -4.222 & 0.375 \\
& LA_NORF-LA_RFH & -1.393 & 0.090 \\
& HA_NORF-HA_RFG & -0.095 & 0.471 \\
& HA_NORF-HA_RFH & -0.807 & 0.240 \\
\hline Oculomotor & LA_NORF-LA_RFG & -0.486 & 0.396 \\
& LA_NORF-LA_RFH & -1.393 & 0.106 \\
& HA_NORF-HA_RFG & -0.295 & 0.416 \\
& HA_NORF-HA_RFH & -1.327 & 0.099 \\
\hline
\end{tabular}

${ }^{*}$ and bold $=p<0.05$ (1-tailed significance).

\subsection{Impact of Usage of Distance Spectacles/Contact Lenses}

We did not find any statistically significant differences between the rest-frame conditions for the VRISE scores of participants who successfully completed the experiment. 
Therefore, we analyzed if there were differences between wearers and non-wearers of distance spectacles. The detailed VRISE results assessed by the SSQ, FMS, and VRNQ questionnaire for all conditions are shown in Tables 15 and 16 for both groups.

Table 15. Subjective VRISE levels assessed by the SSQ and VRNQ (VRISE Section) for participants who had normal vision and did not use distance spectacles or contact lenses.

\begin{tabular}{|c|c|c|c|c|c|c|}
\hline VRISE Scale & Condition & $\mathbf{N}$ & Mean & SD & Min Value & Max Value \\
\hline \multirow[t]{6}{*}{ SSQ Total } & LA_NORF & 15 & 25.18 & 29.23 & 0.00 & 104.72 \\
\hline & LA_RFG & 15 & 29.92 & 33.54 & 0.00 & 119.68 \\
\hline & LA_RFH & 15 & 36.40 & 37.06 & 0.00 & 123.42 \\
\hline & HA_NORF & 15 & 33.66 & 34.86 & 0.00 & 112.20 \\
\hline & HĀ_RFG & 15 & 36.15 & 28.85 & 0.00 & 78.54 \\
\hline & HA_RFH & 15 & 27.93 & 26.55 & 0.00 & 71.06 \\
\hline SSQ & LA_NORF & 15 & 24.13 & 39.54 & 0.00 & 139.20 \\
\hline \multirow[t]{5}{*}{ Disorientation } & LĀ_RFG & 15 & 36.19 & 45.81 & 0.00 & 125.28 \\
\hline & LA_RFH & 15 & 42.69 & 47.20 & 0.00 & 167.04 \\
\hline & HA_NORF & 15 & 38.05 & 40.57 & 0.00 & 125.28 \\
\hline & HA_RFG & 15 & 31.55 & 31.33 & 0.00 & 83.52 \\
\hline & HA_RFH & 15 & 27.84 & 30.22 & 0.00 & 83.52 \\
\hline \multirow[t]{6}{*}{ SSQ Nausea } & LA_NORF & 15 & 27.98 & 27.57 & 0.00 & 95.40 \\
\hline & LA_RFG & 15 & 28.62 & 29.95 & 0.00 & 95.40 \\
\hline & LA_RFH & 15 & 36.25 & 36.27 & 0.00 & 104.94 \\
\hline & HA_NORF & 15 & 35.62 & 41.66 & 0.00 & 124.02 \\
\hline & HĀ_RFG & 15 & 43.24 & 39.48 & 0.00 & 114.48 \\
\hline & HA_RFH & 15 & 30.53 & 35.00 & 0.00 & 95.40 \\
\hline SSQ & LA_NORF & 15 & 15.67 & 23.36 & 0.00 & 90.96 \\
\hline \multirow[t]{5}{*}{ Oculomotor } & LĀ_RFG & 15 & 18.19 & 25.27 & 0.00 & 98.54 \\
\hline & LA_RFH & 15 & 21.73 & 24.79 & 0.00 & 90.96 \\
\hline & HA_NORF & 15 & 19.20 & 20.43 & 0.00 & 68.22 \\
\hline & HA_RFG & 15 & 21.73 & 20.83 & 0.00 & 68.22 \\
\hline & HA_RFH & 15 & 17.18 & 20.75 & 0.00 & 75.80 \\
\hline VRNQ & LA_NORF & 15 & 31.07 & 4.86 & 20 & 35 \\
\hline \multirow[t]{5}{*}{ VRISE scale } & LA_RFG & 14 & 30.43 & 6.20 & 15 & 35 \\
\hline & LA_RFH & 15 & 31.13 & 4.24 & 21 & 35 \\
\hline & HA_NORF & 15 & 30.07 & 5.40 & 20 & 35 \\
\hline & HA_RFG & 15 & 29.60 & 5.82 & 19 & 35 \\
\hline & HA_RFH & 15 & 31.13 & 4.39 & 22 & 35 \\
\hline
\end{tabular}

Table 16. Subjective VRISE levels assessed by the SSQ and VRNQ (VRISE Section) for participants who had normal vision and did use distance spectacles or contact lenses (during the experiment, they were wearing contact lenses).

\begin{tabular}{ccccccc}
\hline VRISE Scale & Condition & N & Mean & SD & Min Value & Max Value \\
\hline SSQ Total & LA_NORF & 9 & 17.04 & 19.44 & 0.00 & 56.10 \\
& LA_RFG & 9 & 19.12 & 22.40 & 0.00 & 71.06 \\
& LA_RFH & 9 & 22.44 & 26.45 & 0.00 & 67.32 \\
& HA_NORF & 9 & 24.93 & 24.31 & 0.00 & 63.58 \\
& HA_RFG & 9 & 18.28 & 19.11 & 0.00 & 52.36 \\
& HA_RFH & 9 & 27.43 & 23.58 & 0.00 & 67.32 \\
\hline Disorientation & LA_NORF & 9 & 17.01 & 20.62 & 0.00 & 55.68 \\
& LA_RFG & 9 & 21.65 & 31.21 & 0.00 & 97.44 \\
& LA_RFH & 9 & 23.20 & 30.34 & 0.00 & 83.52 \\
& HA_NORF & 9 & 32.48 & 36.17 & 0.00 & 83.52 \\
& HA_RFG & 9 & 24.75 & 28.51 & 0.00 & 83.52 \\
& HA_RFH & 9 & 30.93 & 30.95 & 0.00 & 69.60 \\
\hline
\end{tabular}


Table 16. Cont.

\begin{tabular}{ccccccc}
\hline VRISE Scale & Condition & N & Mean & SD & Min Value & Max Value \\
\hline SSQ Nausea & LA_NORF & 9 & 18.02 & 21.03 & 0.00 & 47.70 \\
& LA_RFG & 9 & 18.02 & 20.49 & 0.00 & 47.70 \\
& LA_RFH & 9 & 25.44 & 30.91 & 0.00 & 85.86 \\
& HA_NORF & 9 & 25.44 & 26.13 & 0.00 & 66.78 \\
& HA_RFG & 9 & 14.84 & 18.54 & 0.00 & 47.70 \\
& HA_RFH & 9 & 27.56 & 28.80 & 0.00 & 95.40 \\
\hline SSQ & LA_NORF & 9 & 10.95 & 14.73 & 0.00 & 45.48 \\
Oculomotor & LA_RFG & 9 & 12.63 & 16.08 & 0.00 & 53.06 \\
& LA_RFH & 9 & 12.63 & 15.16 & 0.00 & 45.48 \\
& HA_NORF & 9 & 12.63 & 14.18 & 0.00 & 45.48 \\
& HA_RFG & 9 & 11.79 & 12.63 & 0.00 & 37.90 \\
VRNQ & HA_RFH & 9 & 16.84 & 18.09 & 0.00 & 53.06 \\
\hline VRISE scale & LA_NORF & 9 & 32.56 & 2.60 & 28 & 35 \\
& LA_RFG & 9 & 32.44 & 2.83 & 27 & 35 \\
& LA_RFH & 9 & 33.22 & 2.17 & 30 & 35 \\
& HA_NORF & 9 & 31.56 & 4.13 & 23 & 35 \\
& HA_RFG & 9 & 32.33 & 3.04 & 27 & 35 \\
& HA_RFH & 9 & 31.11 & 3.59 & 26 & 35 \\
\hline
\end{tabular}

The Shapiro-Wilk test showed that the data was not normally distributed. Therefore, we used nonparametric statistical tests. To observe the differences between the combinations of rest-frame conditions for the VRISE scores and examine the hypotheses, the Wilcoxon signed-rank test was used, as presented in Tables 17 and 18.

Table 17. Wilcoxon signed-rank test for VRISE scores between rest-frame conditions for participants who had normal vision and did not use distance spectacles or contact lenses.

\begin{tabular}{cccc}
\hline VRISE Scale & Condition & $\mathbf{Z}$ & $p$ \\
\hline SSQ Total & LA_RFG-LA_RFH & -1.122 & 0.280 \\
& HA_RFG-HA_RFH & -1.963 & 0.054 \\
\hline SSQ & LA_RFG-LA_RFH & -0.821 & 0.441 \\
Disorientation & HA_RFG-HA_RFH & -0.796 & 0.484 \\
\hline SSQ Nausea & LA_RFG-LA_RFH & -0.893 & 0.395 \\
& HA_RFG-HA_RFH & $-\mathbf{2 . 3 6 0}$ & $\mathbf{0 . 0 1 8}$ \\
\hline SSQ & LA_RFG-LA_RFH & -0.732 & 0.510 \\
Oculomotor & HA_RFG-HA_RFH & -1.569 & 0.137 \\
\hline VRNQ & LA_RFG-LA_RFH & 0.000 & 1.000 \\
VRISE scale & HA_RFG-HA_RFH & $\mathbf{- 2 . 1 9 5}$ & $\mathbf{0 . 0 3 1}$ \\
\hline
\end{tabular}

* and bold $=p<0.05$ (2-tailed significance).

Table 18. Wilcoxon signed-rank test for VRISE scores between the rest-frame conditions for participants who had normal vision and did use distance spectacles or contact lenses (during the experiment, they were wearing contact lenses).

\begin{tabular}{cccc}
\hline VRISE Scale & Condition & Z & $p$ \\
\hline SSQ Total & LA_RFG-LA_RFH & -0.632 & 0.656 \\
& HA_RFG-HA_RFH & -1.620 & 0.133 \\
\hline SSQ & LA_RFG-LA_RFH & -0.276 & 1.000 \\
Disorientation & HA_RFG-HA_RFH & -0.816 & 0.750 \\
\hline SSQ Nausea & LA_RFG-LA_RFH & -1.667 & 0.188 \\
& HA_RFG-HA_RFH & $-\mathbf{2 . 2 3 2}$ & $\mathbf{0 . 0 3 1}$ \\
\hline SSQ & LA_RFG-LA_RFH & 0.000 & 1.000 \\
Oculomotor & HA_RFG-HA_RFH & -0.736 & 0.625 \\
\hline VRNQ & LA_RFG-LA_RFH & -1.511 & 0.250 \\
\hline VRISE scale & HA_RFG-HA_RFH & -1.289 & 0.375 \\
\hline
\end{tabular}

* and bold $=p<0.05$ (2-tailed significance). 
Using glasses as the rest-frame in the HA mode of the game did cause elevated VRISE levels compared to the baseball hat as the rest-frame, as assessed by the SSQ Nausea score, as well as by the VRNQ VRISE scale, for participants who had normal vision and did not wear distance spectacles. This supported Hypothesis H2 for the SSQ Nausea subscale and VRNQ-VRISE scale.

In contrast to the users who did not wear distance spectacles, the usage of glasses as the rest-frame in the HA mode of the game, as assessed by the SSQ Nausea subscale, helped reduce the VRISE levels compared to the usage of the baseball hat as the rest-frame. Those users used distance spectacles or contact lenses, but during the experiment, they were wearing contact lenses under the HMD.

\section{Discussion}

In this study, we researched the effects of different types of head-centric rest-frames on Virtual Reality-Induced Symptoms and Effects (VRISE), user experience, presence, and performance in a custom-designed VR game. We used two types of head-centric rest-frames: glasses (RFG) in the central vision and baseball hat (RFH) in the peripheral vision in two different modes of the game: low action (LA) and high action (HA) modes. Compared to the baseball hat, the glasses occupied a larger part of the VR scene and directly occluded the scene of a virtual environment. The glasses were mostly perceived by the participants, whereas a baseball hat was rarely perceived by them. It remains doubtful if a baseball hat will be selected as a rest-frame in the HA mode of the game, where more attention to the game was needed. We found exciting and mixed results, which should have important implications for designing enjoyable VR experiences.

In a low action mode of the game, where participants were walking and slowly rotating in a virtual environment, without any other provocative movements, the impact of both types of head-centric rest-frames was negative on the VRISE level (more sickness). The SSQ Total and all SSQ Subscales scores (Disorientation, Nausea, and Oculomotor) were significantly higher when rest-frames were used, except for the SSQ Nausea scores when rest-frames glasses were used.

In a high action mode, where participants were running and quickly rotating in a virtual environment, with additional provocative movements (jumping), the results were in contrast to the low action mode. Although the mean VRISE scores showed mostly positive effects (fewer VRISE symptoms) when the rest-frames were used, the differences were not significant, so drawing any conclusions was not possible. However, we found a noticeable drop in the SSQ Total and Disorientation maximum scores when the rest-frames were used compared to no rest-frame usage. The maximum values of the SSQ Total and SSQ Disorientation scores in the HA mode of the game with the rest-frames were even lower than in the less provocative LA mode of the game for any of the conditions. We can conclude that using rest-frames in the HA mode of the game influenced the maximum SSQ Total and SSQ Disorientation scores.

Finding subgroups of participants where differences in the mean scores would be significant lead us to subgroups of participants who previously experienced VR technology.

For participants with previous experience with VR technology prior to the experiment, we found that the usage of glasses as rest-frames in the HA mode of the game significantly lowered the SSQ Disorientation scores (less disorientation-related side effects). For the other SSQ scales, lower mean SSQ scores (less sickness) were observed when both types of rest-frames were used, except for the LA_RFG condition for the SSQ Nausea subscale. When using a baseball hat as a rest-frame in the HA mode of the game, the difference was near statistical significance.

We did not find any difference between both types of rest-frames in terms of the VRISE, user experience, presence, and performance. Analyzing the effects of rest-frames on the subgroups of users who did not wear distance spectacles and had normal vision, it was found out that using glasses as a rest-frame in the HA mode of the game did cause elevated VRISE levels for the SSQ Nausea scores and for the VRNQ VRISE scores compared to the 
baseball hat as the rest-frame. In contrast to the users' who did wear distance spectacles usage of glasses as the rest-frame in the HA mode of the game did help with reducing the VRISE levels compared with the usage of the baseball hat as the rest-frame for the SSQ Nausea scores. Those users used distance spectacles or contact lenses, but during the experiment, they were wearing contact lenses under the HMD.

For the participants who wore distance spectacles during the experiment under the HMD, we observed elevated VRISE levels in the LA and HA modes of the game when using glasses as the rest-frames. Elevated VRISE levels were observed with all VRISE questionnaires and their subscales (SSQ, FMS, and the VRNQ-VRISE subscale), but the differences were not statistically significant.

Based on these findings, we do not recommend the usage of glasses as rest-frames for users who are not wearing distance spectacles and are not used to having visible frames of distance spectacles in front of their eyes all of the time.

We did not find any effect of the rest-frame on the user experience for the UEQ Overall and Hedonic quality or for the VRNQ-User Experience scale. The only statistically significant difference found was in the LA mode of the game when using the rest-frame glasses for the UEQ-S Pragmatic Quality, which caused lower user experience compared to the non-usage of rest-frames.

The presence was only affected when rest-frame glasses were used in the LA mode of the game for the SPES Total score and the SPES Self-location and SPES Possible actions subscales. Using the rest-frame glasses in the LA mode of the game did affect the presence negatively (less presence).

Regarding performance (score and time), we did not find any statistically significant differences between the no rest-frame and rest-frame conditions, so we can conclude that the usage of head-centric rest-frames did not affect the performance.

Comparing the modes of the game, we found significantly fewer disorientation symptoms and significantly fewer sickness symptoms assessed by the FMS Questionnaire for the LA mode of the game. For the user experiences, it was found that better experiences were in the HA mode of the game for the UEQ-S Overall scores for the RFG condition, for the UEQ-S Hedonic Quality scores for the NORF and the RFG conditions, and the VRNQ-User Experience scores for all conditions. The UEQ-S Pragmatic Quality subscale data showed that the UEQ-S Pragmatic Quality scores were significantly lower in the HA mode of the game for the NORF condition. The results showed that, although the VRISE levels were higher in the HA mode of the game, the user experience was better, except for the pragmatic quality of the user experience. An impact on presence was found only for the RFG conditions of the game (higher presence) for the SPES Total scores, as well as for the SPES Self-location and SPES Possible Actions subscales

\section{Limitations and Mitigations}

The study results should be validated and applied to other types of contents and performed on other types of head-mounted displays. This experiment was conducted on an Oculus Rift $\mathrm{S}$ head-mounted display with gaming content.

The differences between the low action and high action modes of the game could be argued. Our general intention was to use different modes of the game, low action being slow and "boring" and the high action with highly reactive psychophysiology, so that the participants experienced different VR scenarios to easier examine their responses to VR stimuli. A review of previous studies showed us that the selection of possible actions, speed of translation, and rotations were suitable for assessing the participants' VRISE, user experiences, and presence.

To ensure that the participants adjusted and centered the HMD and picture optimally, the experimenters carefully managed the HMDs.

The sample size was relatively small, especially because of the relatively large dropout rate $(22.73 \%)$, and only completed experiments could be included in the analysis. How- 
ever, the sample size offered adequate statistical power for the performance analyses, as compared to other relevant works and studies.

The locations and number of places where the FMS scores were collected during the gameplay could also be argued. However, given that FMS was collected after finishing each scene of the scenario and before and at the end of each scenario, the selection of locations seemed appropriable.

A limitation related to this study was the sampling of the participants. The majority of the sample was gathered from the University of Ljubljana-Faculty of Electrical Engineering and web pages dedicated to gaming and virtual reality and invitations on social media. Therefore, the majority of participants who attended the experiment were interested in VR technology. Therefore, generalizing the results to other groups should be addressed in future studies.

Another limitation associated with this study was that the increase in the familywise error rate across the reported statistical analyses was not controlled. However, considering related studies, there is a disagreement on whether those multiple comparisons are really necessary, whether the increase of the familywise error rate is important or not, and if the Bonferroni correction is too strict [40-42].

\section{Conclusions and Future Work}

This study examined the effects of head-centric rest-frames in terms of VRISE, user experience, and presence in virtual environments. The study results were somehow mixed, and more future work must be done to research the effects of head-centric rest-frames in more depth. The study showed that head-centric rest-frames are not helpful in terms of VRISE gaming contents where translation and rotation is slow (walking) and there is no vertical movement in the y-axis. In more action gaming contents with fast translation and rotation and vertical movement (jumps), the study showed that head-centric rest-frames are helpful for users who are familiar with virtual reality technology. For all users, there were differences in the mean VRISE levels, but we could not confirm that as statistically significant. Our study also showed that virtual glasses are helpful for users who use distance spectacles but not when the real glasses are worn underneath the HMD. It is obviously disturbing to have "double" glasses when experiencing a virtual environment.

They are not recommended for new users of virtual reality, as we found statistically significant differences with a positive impact on the SSQ Disorientation levels only for participants who had previous experience with VR technology. Additional objects in the virtual environment for users experiencing virtual reality for the first time and are being overwhelmed with new visual information, perception, and immersion are just too much information to be processed by the nervous system. New users of virtual reality should not be exposed to highly provocative content during their first sessions. They should go through the process of adaptation/habituation to reduce VRISE. Incremental exposure and progressively increasing the intensity of stimulations over multiple exposures is a very effective way to reduce motion sickness [19].

Users who are wearing distance spectacles should choose glasses as the rest-frames (and wear contact lenses under the HMD to avoid the effects of "double" glasses). Nonwearers of distance spectacles should choose a baseball hat.

Head-centric rest-frames is a promising solution to be implemented in an application or in a game where there is an emphasis on reducing the VRISE levels. They are easily implemented, and they do not drastically change the design and appearance of the game. We propose the usage of rest-frames as an optional setting to be activated as preferred by the user, based on their personal preferences and experiences.

In future works, the effects of head-centric rest-frames should be performed in more depth, with more participants, as this was a potential limitation of this study. Since restframes can be disturbing for some participants, dynamic rest-frames could be implemented. That means that, in low action contents or while being still, rest-frames should not be visible or be transparent, but in high action contents or when moving or rotating fast, rest-frames 
should be displayed. In our study, rest-frames were tested in the case of smooth artificial locomotion. Besides other types of contents, it should also be tested on other locomotion interfaces in order to compare and analyze the effects of head-centric rest-frames effectively. One interesting idea is also the usage of darkened lenses, which become darker when the action gets more provocative.

Author Contributions: Conceptualization, A.S., M.P. and J.G.; data curation, A.S.; formal analysis, A.S.; investigation, A.S., M.P. and J.G.; methodology, A.S. and J.G.; project administration, J.G.; software, J.G.; supervision, J.G.; validation, A.S., M.P. and J.G.; visualization, A.S.; writing—original draft, A.S.; and writing-review and editing, A.S., M.P. and J.G. All authors have read and agreed to the published version of the manuscript.

Funding: This research received no external funding.

Institutional Review Board Statement: The study that involves human subjects complied with all the relevant national regulations and institutional policies and was conducted in accordance with the Declaration of Helsinki, and has been approved by the author's institutional review board (24 August 2020).

Informed Consent Statement: Informed consent was obtained from all subjects involved in the study.

Data Availability Statement: The data presented in this study are available on request from the corresponding author.

Conflicts of Interest: The authors declare no conflict of interest.

\section{References}

1. LaViola, J.J. A Discussion of Cybersickness in Virtual Environments. SIGCHI Bull. 2000, 32, 47-56. [CrossRef]

2. Nesbitt, K.; Davis, S.; Blackmore, K.; Nalivaiko, E. Correlating Reaction Time and Nausea Measures with Traditional Measures of Cybersickness. Displays 2017, 48, 1-8. [CrossRef]

3. Szpak, A.; Michalski, S.C.; Saredakis, D.; Chen, C.S.; Loetscher, T. Beyond Feeling Sick: The Visual and Cognitive Aftereffects of Virtual Reality. IEEE Access 2019, 7, 130883-130892. [CrossRef]

4. Rebenitsch, L.; Owen, C. Review on Cybersickness in Applications and Visual Displays. Virtual Real. 2016, 20, 101-125. [CrossRef]

5. LaValle, S.M. Virtual Reality; Cambridge University Press: Cambridge, UK, 2017.

6. Davis, S.; Nesbitt, K.; Nalivaiko, E. A Systematic Review of Cybersickness. In Proceedings of the 2014 Conference on Interactive Entertainment-IE2014, Newcastle, NSW, Australia, 2-3 December 2014; ACM Press: New York, NY, USA, 2014; pp. 1-9.

7. Stone Iii, W.B. Psychometric Evaluation of the Simulator Sickness Questionnaire as a Measure of Cybersickness. Ph.D. Thesis, Iowa State University, Ames, IA, USA, 2017; p. 11054762.

8. Stanney, K.; Lawson, B.D.; Rokers, B.; Dennison, M.; Fidopiastis, C.; Stoffregen, T.; Weech, S.; Fulvio, J.M. Identifying Causes of and Solutions for Cybersickness in Immersive Technology: Reformulation of a Research and Development Agenda. Int. J. Hum. Comput. Interact. 2020, 36, 1783-1803. [CrossRef]

9. Somrak, A.; Humar, I.; Hossain, M.S.; Alhamid, M.F.; Hossain, M.A.; Guna, J. Estimating VR Sickness and User Experience Using Different HMD Technologies: An Evaluation Study. Future Gener. Comput. Syst. 2019, 94, 302-316. [CrossRef]

10. Guna, J.; Geršak, G.; Humar, I.; Song, J.; Drnovšek, J.; Pogačnik, M. Influence of Video Content Type on Users' Virtual Reality Sickness Perception and Physiological Response. Future Gener. Comput. Syst. 2019, 91, 263-276. [CrossRef]

11. Davis, S.; Nesbitt, K.; Nalivaiko, E. Comparing the Onset of Cybersickness Using the Oculus Rift and Two Virtual Roller Coasters. In Proceedings of the 11th Australasian Conference on Interactive Entertainment, Sydney, Australia, 27-30 January 2015; Volume 167, p. 12.

12. Rebenitsch, L.R. Cybersickness Prioritization and Modeling; Michigan State University: East Lansing, MI, USA, 2015.

13. Prothero, J.D.; Parker, D.E. A Unified Approach to Presence and Motion Sickness. In Virtual and Adaptive Environments: Applications, Implications, and Human Performance Issues; CRC Press: Boca Raton, FL, USA, 2003; pp. 47-66.

14. Prothero, J.D. The Role of Rest Frames in Vection, Presence and Motion Sickness; University of Washington: Seattle, WA, USA, 1998.

15. Cao, Z.; Jerald, J.; Kopper, R. Visually-Induced Motion Sickness Reduction via Static and Dynamic Rest Frames. In Proceedings of the 2018 IEEE Conference on Virtual Reality and 3D User Interfaces (VR), Reutlingen, Germany, 18-22 March 2018; IEEE: New York, NY, USA, 2018; pp. 105-112.

16. Chang, E.; Hwang, I.; Jeon, H.; Chun, Y.; Kim, H.T.; Park, C. Effects of Rest Frames on Cybersickness and Oscillatory Brain Activity. In Proceedings of the 2013 International Winter Workshop on Brain-Computer Interface (BCI), Gangwo, Korea, 18-20 February 2013; IEEE: New York, NY, USA, 2013; pp. 62-64.

17. Nguyen-Vo, T.; Riecke, B.E.; Stuerzlinger, W. Simulated Reference Frame: A Cost-Effective Solution to Improve Spatial Orientation in VR. In Proceedings of the 2018 IEEE Conference on Virtual Reality and 3D User Interfaces (VR), Reutlingen, Germany, 18-22 March 2018; IEEE: New York, NY, USA, 2018; pp. 415-422. 
18. Wienrich, C.; Weidner, C.K.; Schatto, C.; Obremski, D.; Israel, J.H. A Virtual Nose as a Rest-Frame-The Impact on Simulator Sickness and Game Experience. In Proceedings of the 2018 10th International Conference on Virtual Worlds and Games for Serious Applications (VS-Games), Wurzburg, Germany, 5-7 September 2018; IEEE: New York, NY, USA, 2018; pp. 1-8.

19. Jerald, J. The VR Book: Human-Centered Design for Virtual Reality, 1st ed.; ACM Books; ACM, Association for Computing Machinery: New York, NY, USA, 2016; ISBN 978-1-970001-12-9.

20. Oman, C.M. Motion Sickness: A Synthesis and Evaluation of the Sensory Conflict Theory. Can. J. Physiol. Pharmacol. 1990, 68, 294-303. [CrossRef] [PubMed]

21. Hale, K.S.; Stanney, K.M.; Wogalter, M.; Sears, A.; Jacko, J.A. Handbook of Virtual Environments: Design, Implementation, and Applications, 2nd ed.; CRC Press: Boca Raton, FL, USA, 2015.

22. Lin, J.J.-W.; Abi-Rached, H.; Kim, D.-H.; Parker, D.E.; Furness, T.A. A “Natural” Independent Visual Background Reduced Simulator Sickness. In Proceedings of the Human Factors and Ergonomics Society Annual Meeting, Baltimore, MD, USA, 29 September-4 October 2002; Volume 46, pp. 2124-2128.

23. Riecke, B.E.; Schulte-Pelkum, J.; Caniard, F.; Bülthoff, H.H. Towards Lean and Elegant Self-Motion Simulation in Virtual Reality. In Proceedings of the IEEE Proceedings, VR 2005, Virtual Reality 2005, Bonn, Germany, 12-16 March 2005; pp. 131-138.

24. Duh, H.B.-L.; Parker, D.E.; Furness, T.A. An “Independent Visual Background” Reduced Balance Disturbance Evoked by Visual Scene Motion: Implication for Alleviating Simulator Sickness. In Proceedings of the SIGCHI Conference on Human Factors in Computing Systems, Seattle, WA, USA, 31 March-4 April 2001; pp. 85-89.

25. Lin, J.J.W.; Abi-Rached, H.; Lahav, M. Virtual Guiding Avatar: An Effective Procedure to Reduce Simulator Sickness in Virtual Environments. In Proceedings of the 2004 Conference on Human Factors in Computing Systems-CHI '04, Vienna, Austria, 24-29 April 2004; ACM Press: New York, NY, USA, 2004; pp. 719-726.

26. Prothero, J.D.; Parker, D.E. The Use of an Independent Visual Background to Reduce Simulator Side-Effects. Aviat. Space Environ. Med. 1999, 70 Pt 1, 277-283.

27. Kennedy, R.S.; Lane, N.E.; Berbaum, K.S.; Lilienthal, M.G. Simulator Sickness Questionnaire: An Enhanced Method for Quantifying Simulator Sickness. Int. J. Aviat. Psychol. 1993, 3, 203-220. [CrossRef]

28. Keshavarz, B.; Hecht, H. Validating an Efficient Method to Quantify Motion Sickness. Hum. Factors 2011, 53, 415-426. [CrossRef] [PubMed]

29. Kourtesis, P.; Collina, S.; Doumas, L.A.A.; MacPherson, S.E. Validation of the Virtual Reality Neuroscience Questionnaire: Maximum Duration of Immersive Virtual Reality Sessions without the Presence of Pertinent Adverse Symptomatology. Front. Hum. Neurosci. 2019, 13, 417. [CrossRef] [PubMed]

30. Hartmann, T.; Wirth, W.; Schramm, H.; Klimmt, C.; Vorderer, P.; Gysbers, A.; Böcking, S.; Ravaja, N.; Laarni, J.; Saari, T.; et al. The Spatial Presence Experience Scale (SPES): A Short Self-Report Measure for Diverse Media Settings. J. Media Psychol. 2016, 28, 1-15. [CrossRef]

31. Schrepp, M.; Hinderks, A.; Thomaschewski, J. Design and Evaluation of a Short Version of the User Experience Questionnaire (UEQ-S). IJIMAI 2017, 4, 103. [CrossRef]

32. Ijsselsteijn, W.; Riva, G. Being There: The Experience of Presence in Mediated Environments. In Being There: Concepts, Effects and Measurements of User Presence in Synthetic Environments; IOS Press: Amsterdam, The Netherlands, 2003; 14p.

33. Veale, J.F. Edinburgh Handedness Inventory-Short Form: A Revised Version Based on Confirmatory Factor Analysis. Laterality Asymmetries Body Brain Cogn. 2014, 19, 164-177. [CrossRef] [PubMed]

34. Witmer, B.G.; Singer, M.J. Measuring Presence in Virtual Environments: A Presence Questionnaire. Presence Teleoperators Virtual Environ. 1998, 7, 225-240. [CrossRef]

35. Lovik, A.; Verbeke, G.; Molenberghs, G. BFI-10: A Very Short Personality Test to Measure the Big Five Factors. In Proceedings of the Leuven Statistics Days, Leuven, Belgium, 20-21 October 2016. [CrossRef]

36. Saredakis, D.; Szpak, A.; Birckhead, B.; Keage, H.A.D.; Rizzo, A.; Loetscher, T. Factors Associated with Virtual Reality Sickness in Head-Mounted Displays: A Systematic Review and Meta-Analysis. Front. Hum. Neurosci. 2020, 14, 96. [CrossRef] [PubMed]

37. Tableau Prep: A New Data Preparation Solution. Available online: https://www.tableau.com/products/prep (accessed on 15 December 2020).

38. IBM SPSS Software. Available online: https://www.ibm.com/analytics/spss-statistics-software (accessed on 12 December 2020).

39. RStudio-Open Source and Professional Software for Data Science Teams. Available online: https://rstudio.com/ (accessed on 12 December 2020).

40. Perneger, T.V. What's Wrong with Bonferroni Adjustments. BMJ 1998, 316, 1236-1238. [CrossRef] [PubMed]

41. Naeem, S. To Bonferroni or Not to Bonferroni: When and How Are the Questions. Bull. Ecol. Soc. Am. 2000, 81, $246-248$.

42. Nakagawa, S. A Farewell to Bonferroni: The Problems of Low Statistical Power and Publication Bias. Behav. Ecol. 2004, 15, 1044-1045. [CrossRef] 